\title{
Nanoscale origin and evolution of kinetically induced defects in carbon spheres
}

\author{
Sanket A. Deshmukh ${ }^{1}$, Badri Narayanan ${ }^{1}$, Ganesh Kamath ${ }^{2}$, Vilas G. Pol ${ }^{3}$, Jianguo Wen ${ }^{4}$, Dean J. \\ Miller, ${ }^{4}$ and Subramanian K.R.S. Sankaranarayanan ${ }^{1} *$ \\ ${ }^{1}$ Center for Nanoscale Materials, Argonne National Laboratory, Lemont, IL 60439, USA \\ ${ }^{2}$ Department of Chemistry, University of Missouri-Columbia, Columbia 65211, USA \\ ${ }^{3}$ School of Chemical Engineering, Purdue University, West Lafayette 47907, USA \\ ${ }^{4}$ Electron Microscopy Center-Center for Nanoscale Materials, Argonne National Laboratory, Lemont, IL 60439, \\ USA
}

\begin{abstract}
Synthesis of carbon nanostructures with controlled defect distribution holds tremendous promise in engineering novel materials for specific device applications and catalysis. Carbon spheres (CS) can be synthesized by controlled thermal pyrolysis of polyethylene (PE) via a series of self-generated pressure reactions. Here, we show that point defect distribution in these CS can be tailored by controlling the kinetics during this synthesis procedure. Using accelerated molecular dynamics, we demonstrate that cooling kinetics during synthesis exercises significant control over the reaction pathways, and consequently, over nucleation and growth of carbon nanostructures. The long-range order of these nanostructures is strongly influenced by the cooling rate. At slower rates $<1.4 \mathrm{~K} / \mathrm{ps}$, well-ordered carbon onions form with very few defects, while at higher rates $>60 \mathrm{~K} / \mathrm{ps}$, amorphous carbon with 8-10 membered rings reminiscent of as-quenched carbon melts are seen. In the intermediate regime, the distribution of the defects can be tuned with excellent control. These findings are corroborated by electron microscopy observations of defect formations in synthesized CS. SEM and TEM on CS when cooled from $\sim 973 \mathrm{~K}\left(700^{\circ} \mathrm{C}\right)$ to room temperature indicate smooth surface
\end{abstract}

Corresponding author: sanket@anl.gov

Corresponding author: skrssank@anl.gov

(C) 2015. This manuscript version is made available under the Elsevier user license http://www.elsevier.com/open-access/userlicense/1.0/ 
morphologies with a highly defective structure. In contrast, when cooled from $\sim 3073 \mathrm{~K}$ $\left(2800^{\circ} \mathrm{C}\right)$, rough surfaces with pronounced 6-membered rings graphitic ordering are observed.

\section{Introduction}

Atomic scale defects in carbon nanostructures can significantly alter their physical, chemical, mechanical, magnetic and electronic properties [1-3]. Intrinsic point defects (vacancy-type defects) have a st rong influence on the reactivity of carbon nanostructures, making these defective structures as prospective catalysts [4]. Stone-Wales (SW) defects and reconstructed vacancies have been shown to locally change the density of $\pi$-electrons and increase reactivity [5]. Point defects such as vacancies tend to reduce the Young's modulus and tensile strength [6]. These defects also strongly influence the electronic properties via local rehybridization of $\sigma$ - and $\pi$ - orbitals in the vicinity of structural defects $[7,8]$. Apart from point defects, extended line defects in carbon nanostructures have been reported to alter atomic charge distribution as well as electronic spin [9]. Similarly, defects in the graphitic network such as vacancies, interstitials and ad-atoms have local magnetic moments and can give rise to magnetic ordering $[10,11]$. Evidently, a fundamental understanding of these defects is crucial to design carbon nanostructures with target properties [12]; in particular, their formation dynamics and evolution during the synthesis and processing of carbon nanostructures.

Several types of defects are possible in carbon allotropes (graphene, nanotubes, onions and fullerenes), including edges, grain boundaries, vacancies implanted atoms, and those associated with a change in hybridization state of carbon, e.g., $s p^{2}$ to $s p^{3}[5,9,13]$. These defects can be broadly classified as (a) intrinsic when the graphitic order is perturbed without any foreign atoms, and (b) extrinsic if induced by i mpurities or dopants [14]. The intrinsic defects may have different dimensionalities; point defects such as vacancies or interstitials are 
zero-dimensional, dislocations are one-dimensional, grain boundaries or stacking faults are two dimensional, whereas inclusions and voids are three-dimensional defects [14]. As aforementioned, each of these defect types strongly influences the physical and electronic properties of nanostructures. A precise control over the location of these defects and their arrangement into ordered and extended structures can allow for design of carbon nanostructures with novel properties [15].

One can clearly envisage several diversified applications of nanocarbon materials by taking advantage of intrinsic and extrinsic defects, which can be induced locally during their synthesis and processing $[16,17]$. A lot of recent research has therefore been focused on achieving controlled synthesis of defects in graphene and other carbon nanostructures.[14, 17, 18] For instance, it has been shown that energetic particles such as electrons and ions can be used to induce polymorphic atomic defects in carbon nanostructures as a result of irradiation induced knock-on atom displacements [18, 19]. In addition, several chemical methods (e.g., chemical vapor deposition) have been employed to induce intrinsic defects with non-hexagonal bonding geometries [20]. Extrinsic defects can also be incorporated via doping agents [14]. In these synthesis methods, it is commonly observed that a high deposition temperature facilitates relaxation towards thermal equilibrium and rapid annealing of defects, while low temperature growth invariably leads to higher amount of defects.

Recently, we introduced a unique route to synthesize carbon nanomaterials using controlled thermal pyrolysis, in which one or more precursors such as polyethylene wastes (PE) are decomposed at elevated temperatures via a series of self-generated pressure reactions [21-24]. In this technique, precursors such as PE are heated to high temperatures to attain "supercritical phases" which are allowed to interact in a cl osed system [21, 25]. The 
thermodynamic and kinetic stabilities of the resultant product phases during the nucleation and growth processes yield new carbon materials (e.g. CS, cobalt-encapsulated carbon nanotubes, metallic core-carbon shell, sausage like carbon bodies, and egg-like CS etc.) in nano-to-micro dimensions [22, 24, 26-30]. These CS are expected to possess interesting electronic, optical, and other physical properties. For example, these CS have been shown to significantly improve performance when used as lubricants in tribological applications and as anode materials in battery applications $[12,24]$. We note that the synthesis protocol entails numerous dynamical processes involving a multitude of interfacial reactions, nucleation, and microstructural evolution [22]. Naturally, therefore, the material defect chemistry is a strong function of atomistic transport phenomena across a growing interface, which in turn is influenced by the annealing or cooling dynamics.

Here, we demonstrate using reactive molecular dynamics (MD) simulations that controlling the kinetics of crystallization of amorphous carbon melts (obtained from pyrolysis of PE) allows for systematically altering the defect evolution and distribution in carbon nanoallotropes. The nature and dynamics of crystallization in the ultrafast cooling regime is studied by analyzing the MD simulation trajectories. We track the evolution of graphitic order, intrinsic defects, as well as long-range and short-range order as a function of varying cooling rates. The starting amorphous carbon structures are obtained by hi gh temperature thermal pyrolysis of PE; various dehydrogenated fractions are carefully evaluated to assess the effect of residual hydrogen on the defect chemistry and formation in the final carbon nanostructures. Comparisons to experiments are made where possible. In particular, experiments are used to understand the effect of cooling on the formation of microstructural defects whereas atomistic simulations are used to understand the evolution of intrinsic and topological defects during the 
synthesis of carbon structures from polyethylene wastes. Our study demonstrates that new families of nanocarbon allotropes with controlled defect distribution can be formed from amorphous carbon by kinetically tuning the crystallization dynamics.

\section{Methods}

\subsection{Experiments}

\section{(2.1.1) Synthesis of spherical carbon particles}

The completely spherical carbon particles were synthesized by the controlled thermal decomposition of either organic compound such as trashed polyethylene terephthalate [31], polyethylene bags [24] or mesitylene $\left(\mathrm{C}_{9} \mathrm{H}_{12}\right)$ [26]. The reactor is specially designed using Haynes 230 alloy to hold pressure up to 2000 psi and temperature could be as high as $\geq \sim 1073$ $\mathrm{K}\left(\geq 800^{\circ} \mathrm{C}\right)$. To release excess overpressure built in autoclave, the pressure release valve was also connected. Since the cracking/dissociation of hydrocarbon reaction occurs above $\sim 773 \mathrm{~K}$ $\left(500^{\circ} \mathrm{C}\right),[32]$ most of such reactions are carried out at $\sim 973 \mathrm{~K}\left(700^{\circ} \mathrm{C}\right)$ with a heating rate of $20{ }^{\circ} \mathrm{C} / \mathrm{min}$. The dissociation of hydrocarbon occurs in a short time and thus the reactor was held at $\sim 973 \mathrm{~K}\left(700{ }^{\circ} \mathrm{C}\right.$ ) for $5-10 \mathrm{~min}$, which usually generates pressure of less than $1000 \mathrm{psi}$ at elevated temperature. Thereafter, the heated reactor was cooled naturally to room temperature. The procedure was repeated by synthesizing at $\sim 3073 \mathrm{~K}\left(2800^{\circ} \mathrm{C}\right)$ and subsequently cooling the sample to room temperature. The typical yield of dry, CS was $40-45$ wt. \%. TEM characterization was preformed on the Argonne Aberration-Corrected TEM (ACAT) operated at $80 \mathrm{kV}$ to minimize electron beam-damage to the sample [33]. 


\subsection{Computational details}

\section{(2.2.1) Simulation Set-up}

In the first step, we carried out atomistic simulations of thermal decomposition of PE chains by us ing second-generation reactive empirical bond order (REBO) potential [34, 35]. This potential allows for dynamic breakage and formation of chemical bonds in a variety of hydrocarbons that are likely to form during the thermal decomposition and quenching of hydrocarbon precursors. The decomposition of PE was studied by randomly placing 28 chains of PE consisting of 100 monomer (100-mer) units in a simulation cell with dimensions $(80 \AA \mathrm{x}$ $80 \AA \AA$ x $80 \AA$ ). The ends of each individual PE chain were terminated with hydrogen atoms. The initial equilibration of the PE chains was carried out at room temperature $(\sim 300 \mathrm{~K})$ by performing simulations in a canonical ensemble for 500 ps with a 1 fs timestep [36].

Thermal decomposition of PE was carried out via temperature accelerated reactive molecular dynamics (TARMD) runs in LAMMPS by simulating the systems at temperatures as high as $7000 \mathrm{~K}$ [37]. Our focus here was to completely decompose the polyethylene (PE) and remove the free hydrogen atoms within the MD time scales. Hence, $7000 \mathrm{~K}$ was chosen as a reference temperature as it is sufficiently higher than the melting point of PE and carbon end product. Thus, TARMD technique enables us to explore the degradation mechanism of polymer chains within the time scales accessible to MD simulations. In a typical chemical reaction at elevated temperatures, the hydrogen atoms from the dissociated hydrocarbons escape the system through a free surface or a gas-liquid interface $[22,24]$. In order to emulate experimental conditions of gas-liquid phase separation and decomposition of the polymer in TARMD simulations, it is very important to have an accurate and efficient hydrogen removal scheme. Experimentally, the carbon-hydrogen $(\mathrm{C}-\mathrm{H})$ and hydrogen-hydrogen $(\mathrm{H}-\mathrm{H})$ bond 
lengths are reported to be $1.09 \AA$ and $0.74 \AA$, respectively; these bond lengths are accurately reproduced by the REBO potential $[38,39]$. In our simulations, we identified the hydrogen atoms to be removed (either as free atomic hydrogen or as $\mathrm{H}_{2}$ dimer) from the simulation cell using distance criteria. Specifically, we monitored the distance between carbon and hydrogen atoms every 1 ps (Note, we verified that an increased frequency of monitoring distances does not impact the results significantly). If the separation between a previously bonded C-H pair is $\geq 1.1 \AA$, then the $\mathrm{C}-\mathrm{H}$ bond was assumed to be broken and the respective $\mathrm{H}$ atom was removed from the simulation cell. This is certainly reasonable since the bond cut-off distance in REBO potential is $1.1 \AA$. Similarly, if two $\mathrm{H}$ atoms are $\leq 0.75 \AA$ apart, we considered them to have formed a $\mathrm{H}_{2}$ dimer; we removed the dimer to simulate loss of hydrogen gas.

\section{(2.2.2) Effect of cooling rate on the crystallization dynamics of carbon nanostructures}

We systematically explored the effect of annealing schedule or cooling rate on the crystallization behavior of carbon melts. To accomplish this, we first equilibrate PE chains configuration at room temperature. The equilibrated system was then dehydrogenated by heating up to $\sim 7000 \mathrm{~K}$ over a time-period of $100 \mathrm{ps}$ with a $0.0001 \mathrm{fs}$ time step. We then performed a series of calculations in which the cooling rates were systematically varied to evaluate the effect of cooling on the morphology and defect evolution in the carbon nanostructures. Note, our primary interest lies in the structures that form in the initial stages (nucleation and growth) upon pyrolysis of PE and subsequent cooling. Within these timescales (of the order of several ns) accessible to our MD simulations, the carbon structures, which form possess size (largest dimension) of the order of $<10 \mathrm{~nm}$.

In the first set of simulations, we employed completely dehydrogenated samples. These samples were cooled from $7000 \mathrm{~K}$ to room temperature i.e. $300 \mathrm{~K}$ at varying rates. Five 
different cooling rates were employed in the present study: $67,13.4,6.7,3.35$, and $1.34 \mathrm{~K} / \mathrm{ps}$, respectively. For each of these cycles, we monitored the structure and dynamical evolution of the carbon end product.

In the second set of simulations, we studied the effect of residual hydrogen content on the morphology of carbon nanostructures. At $7000 \mathrm{~K}$, when the PE chains undergo rapid dehydrogenation, the simulation cell was scanned every $1 \mathrm{ps}$ for the presence of free hydrogen or a dimer of hydrogen based on the bond cut-off defined by the REBO potential, as described in Sec. IIB (a). By systematically removing the hydrogen from the simulation cell in this manner, we were able to generate partially dehydrogenated configurations of PE with $0.1,0.5$, 10 , and $20 \%$ residual hydrogen atom concentrations. Each of these was then subjected to similar cooling protocols as described above.

\section{Results and Discussion}

\subsection{Experiments and characterization to reveal kinetic effects on carbon structures}

The synthesis process was used to synthesize carbon structures, which were subjected to two different heating-cooling protocols. SEM and high-resolution TEM (HRTEM) were used to characterize the effects of temperature on the microstructure evolution and defect formation in these structures. Figure 1 shows different surface morphologies observed for the spherical carbon particles (SCPs) obtained by cooling from different initial temperatures (973 $\mathrm{K}$ and $3073 \mathrm{~K})$ to ambient temperature. Note that in both these cases i.e. $\sim 973 \mathrm{~K}\left(700{ }^{\circ} \mathrm{C}\right)$ and $\sim 3073 \mathrm{~K}\left(2800^{\circ} \mathrm{C}\right)$, the systems were allowed to cool naturally to room temperature. Both SEM (Figure 1a and Figure 1d) and low-magnification TEM (the inset in Figure 1 (b) and 1 (e)) show that SCPs cooled from $\sim 973 \mathrm{~K}\left(700{ }^{\circ} \mathrm{C}\right)$ to room temperature have smoother and more uniform surface morphology compared with SCPs cooled from $\sim 3073 \mathrm{~K}\left(2800{ }^{\circ} \mathrm{C}\right)$ to room 
temperature. Figure 1 (b) and 1 (c) show HRTEM images of surface atomic structures for SCPS cooled from $\sim 973 \mathrm{~K}\left(700^{\circ} \mathrm{C}\right)$. Many single irregularly curved layers can be observed at the surface, indicating surface carbon is in the form of graphene with many defects, similar to random or glass-like carbon structures. In contrast, HRTEM images (Figure 1 (e) and 1 (f)) of surface atomic structures for SCPs cooled from $\sim 3073 \mathrm{~K}\left(2800{ }^{\circ} \mathrm{C}\right)$ show totally different surface morphology, exhibiting a well-defined wave-like graphitic structure. Such wave-like graphitic structure of carbon atoms is called as concentric (i.e. parallel to the surface) nanometric texture $[40,41]$. Such an arrangement of carbon atoms can be found in carbon black where carbon atoms are oriented in concentric manner with very little difference in the arrangement of each layer. The concentric texture in spherical bodies is the result of liquid/gas -or solid/gas interface between two different phases [2]. Similar to carbon blacks, the CS produced in present study under self-generated pressure might obey the droplet mechanism [42]. According to the droplet mechanism, the interface between liquid and gas (at $700{ }^{\circ} \mathrm{C}$ synthesis temperature) has reasonably large interfacial energy. In such a scenario, nanometric texture plays a crucial role to minimize surface energy of the particles. In particular, it has been reported that such surface energy minimization can be achieved by exposing the crystalline basal planes of basic structural units to the surroundings; this, in turn, leads to concentric texture $[41,42]$.

It is worth noting that the structure and morphology of the CS observed in our experiments (Fig. 1) are consistent with previous reports, e.g., the anthracites with $\mathrm{C}$ content of 93.9\% and 94.3\% studied by Rosalind Franklin [43]. Specifically, the X-ray powder diffraction patterns of the anthracites heated to temperatures between $\sim 1273 \mathrm{~K}\left(\sim 1000{ }^{\circ} \mathrm{C}\right)$ and $\sim 2273 \mathrm{~K}$ $\left(\sim 2000{ }^{\circ} \mathrm{C}\right)$ is similar to those of the non-graphitizing carbons. In particular, it was found that 
below $\sim 2273 \mathrm{~K}\left(\sim 2000{ }^{\circ} \mathrm{C}\right)$ anthracites form strongly cross-linked and highly porous carbons which greatly resemble the non-graphitizing carbons. On the other hand, at higher temperatures, the cross-linking breaks down; consequently, the near-parallelism of neighboring crystallites causes rapid crystallite growth leading to highly graphitic carbons. The anthracites heated to temperatures above $\sim 2773 \mathrm{~K}\left(\sim 2500{ }^{\circ} \mathrm{C}\right)$ were more highly graphitized [43] than any other graphitizing carbon investigated in previous study.

Note that traditionally CS are synthesized by a carbonization (pyrolysis) process, in which the organic precursors, e.g., hydrocarbon gases, polymers etc. are thermally converted to inorganic solid carbonaceous material in an inert atmosphere [40, 41, 44]. In these synthesis routes, the extent of graphitization, carbon content, order, structure and morphology of the final carbon architecture is dictated by the competition between interfacial energies at the solid/liquid, solid/gas, and liquid/gas interfaces [41]. The relative ordering of these interfacial energies is mainly dependent on the chemical nature of the precursors, and the interfacial chemical reactions. In addition to these from these thermodynamic considerations, the structure/morphology of the formed CS crucially depends on the heat treatment procedures, which control the kinetics of the CS formation processes [44]. For instance, disordered glasslike CS are formed during solid carbonization of organic precursors. On the other hand, chemical vapor deposition methods, owing to sufficient control over kinetics, have been employed to produce CS with pronounced graphitic ordering [41, 44]. Our experiments at extremely high cooling rates, such as those obtained by cooling from $\sim 973 \mathrm{~K}$ to ambient conditions, we obtain a highly disordered CS that closely resembles the glass-like carbon formed during traditional carbonization processes. On the other hand, at slow cooling rates (i.e., cooled from $\sim 3073 \mathrm{~K}$ ), CS with long range graphitic ordering is obtained. These CS 
possess an onion like texture, which consists of numerous graphitic hollow spheres arranged concentrically in a layer-by-layer manner [41]. The structure and morphology of these wellordered CS are reminiscent of carbon blacks with concentric textures, whose structure has been reported to be governed by the droplet mechanism [42]; this mechanism indicates that minimization of surface energies can be achieved via exposing the basal planes of basic structural units (i.e., graphitic rings) to the surrounding, which in turn leads to concentric texture.[42] Similarly, mesocarbon microbeads (upto micron sizes) possessing radial texture with high graphite-like ordering have also been synthesized from mesoscale pitches via heattreatments at temperatures $>2773 \mathrm{~K}[41,45]$.

Based on these results, it is clear that the synthesis protocol, the heat treatment schedules, and the kinetics of the cooling strongly influence the structure and morphology of the final CS.. The temperature profile during the cooling stage was calculated via finite element calculations (see supporting info for details). We note that in the experiments, the cooling rates in both the cases undergo a continuous change with time. As seen in Figure S1, we can observe that the initial rate of cooling is faster which is followed by a slower cooling rate down to room temperature. In the case of $\sim 3073 \mathrm{~K}\left(2800{ }^{\circ} \mathrm{C}\right)$, the system resides for much longer times at the high temperatures compared to the system, which is cooled to $\sim 973 \mathrm{~K}$ $\left(700{ }^{\circ} \mathrm{C}\right)$. Furthermore, if we compare the cooling rates in the $\sim 973 \mathrm{~K}\left(700{ }^{\circ} \mathrm{C}\right)$ to room temperature regime for the two cases, it can be seen that the cooling rate for system cooling from peak temperature of $\sim 3073 \mathrm{~K}\left(2800{ }^{\circ} \mathrm{C}\right)$ is slower compared to the system cooling from peak temperature of $\sim 973 \mathrm{~K}\left(700{ }^{\circ} \mathrm{C}\right)$. The pronounced ordering in SCPs synthesized at elevated temperatures can thus be attributed to the fast kinetics that facilitates rearrangement of 
atoms and consequent annealing of defects. Such a relaxation is hindered at low temperatures $\left(\sim 973 \mathrm{~K}\left(700{ }^{\circ} \mathrm{C}\right)\right)$ due to poor mobility of the atoms leading to a kinetically frustrated amorphous structure.
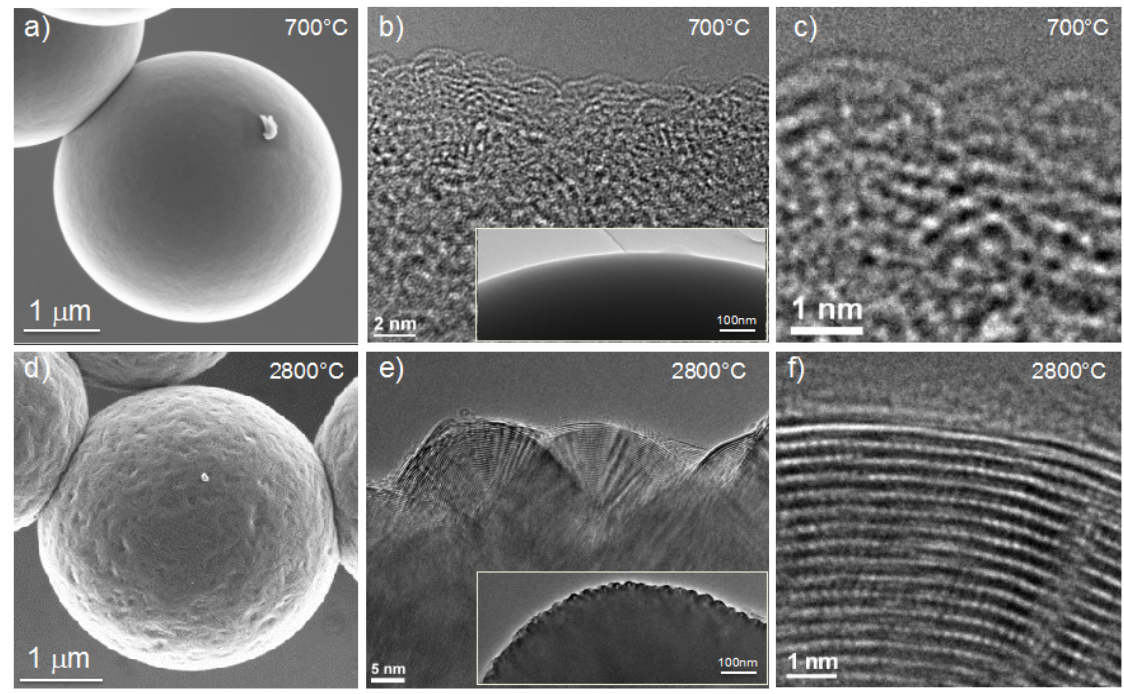

Figure 1. SEM and TEM images showing different surface morphologies of spherical carbon particles (SCP). a) SEM images, b) High-resolution TEM (HRTEM) images, and c) enlarged HRTEM images of SCPs cooled from $\sim 973 \mathrm{~K}\left(70{ }^{\circ} \mathrm{C}\right)$. d) SEM images, e) HRTEM images, and f) Enlarged HRTEM images of SCPs cooled from $~ 3073 \mathrm{~K}$ (2800 $\left.{ }^{\circ} \mathrm{C}\right)$. Note, the SCPs were allowed to cool naturally to room temperature. SCPs cooled from $\sim 973 \mathrm{~K}\left(700{ }^{\circ} \mathrm{C}\right)$ have smooth surfaces with defected curved graphene structures at surfaces. In contrast, SCPs cooled from $\sim 3073 \mathrm{~K}\left(2800{ }^{\circ} \mathrm{C}\right)$ have rough surfaces with curved graphitic structures at surfaces. The lattice spacing shown in $\mathrm{f}$ ) is about $0.33 \mathrm{~nm}$ and $0.2 \mathrm{~nm}$ along and perpendicular to graphitic planes, respectively.

3.2 MD simulations to systematically explore kinetic effects on defect formation in carbon structures

Our microscopy experiments indicate that the crystallization behavior and defect evolution in carbon structures are strongly influenced by the kinetics of the synthesis protocol. Qualitatively based on the residence time and the cooling profile, it can be said that the simulated low cooling rate resemble the natural cooling from higher temperature of $\sim 3073 \mathrm{~K}$ $\left(2800{ }^{\circ} \mathrm{C}\right)$ whereas the simulated high cooling rates resemble natural cooling from $\sim 973 \mathrm{~K}$ $\left(700{ }^{\circ} \mathrm{C}\right)$. To clearly delineate the kinetic effects on the formation and evolving structure of the carbon nuclei, it is essential to have a p recise control on the cooling rates. Atomistic 
simulations allow us to exercise that control in that we can systematically study the dynamical evolution of the system under various constant-cooling rates and thereby evaluate the kinetic effect on the structural and dynamical evolution. Motivated by the kinetic effects seen experimentally on the structure of the CS (Figure 1), we have systematically maintained different constant cooling rates starting from an initial high temperature of $7000 \mathrm{~K}$. The higher cooling rates ensure that the system spends less time at higher temperatures whereas at lower cooling rates, the system resides for a longer time at higher temperatures. Note, that the slow cooling rate qualitatively would be similar to the system cooling from $\sim 3073 \mathrm{~K}\left(2800{ }^{\circ} \mathrm{C}\right)$ and high cooling rate would be akin to that cooling from $\sim 973 \mathrm{~K}\left(700^{\circ} \mathrm{C}\right)$.

In addition, the morphological differences can be characterized (ordered vs. disordered) by using the advanced experimental techniques, however, the atomistic scale defects in the CS are difficult to probe experimentally. Atomic scale defects in carbon nanostructures can significantly alter their physical, chemical, mechanical, magnetic and electronic properties. For example, intrinsic point defects (vacancy-type defects) have a strong influence on the reactivity of carbon nanostructures [46, 47]. In addition, topological defects such as Stone-Wales and reconstructed vacancies have been shown to locally change the density of $\square$-electrons and increase reactivity [48, 49]. Owing to the significant impact of atomistic defects on the observed properties, a fundamental understanding of these defects is crucial to design carbon nanostructures. It is non-trivial experimentally to get the distribution and origin of such vacancies at the nanoscale in structures such as CS. Atomistic simulations provide a viable alternative to probe the origin of such topological defects during the early stages of nucleation and growth of CS. Therefore, to gain further insights into this kinetic dependence of crystallization dynamics and explore kinetic pathways to control defect formation in carbon 
structures, we performed a series of MD simulations with systematically varying cooling rate. These simulations have allowed us to understand the spatio-temporal distribution of defects and the influence of cooling kinetics on the defect formation and evolution in during early stages of nucleation and growth of CS.

\section{(3.2.1) Dynamics of crystallization of carbon nanostructures from dehydrogenated PE}

We first evaluate the effect of the various cooling protocols on the kinetics of crystallization of amorphous carbon melts i.e. completely dehydrogenated configurations $(\sim 0.1 \%$ residual hydrogen). Figure 2 shows the variation in the configurational energy of the system as a function of the cooling temperature. Over the range of cooling rates simulated, the configurational energy decreases with temperature $\left(0.3-0.6 \times 10^{3} \mathrm{eV}\right.$ for the entire system). The temperature range over which the change occurs is $\sim 4000-5000 \mathrm{~K}$ and is a function of the cooling rate; slower cooling rates of $\sim 1.34 \mathrm{~K} / \mathrm{ps}$ results in a more significant change in slope than in the case of faster cooling rates such as $67 \mathrm{~K} / \mathrm{ps}$.

It is interesting to note that the nature of the phase transition from amorphous melt to solid carbon nanostructures appears to be more continuous (or quasi-continuous), especially at faster cooling rates. In the case of a first-order transition, one would expect a sharp drop or discontinuity in the configurational energy curve. This would correspond to the crystallization of the carbon nanostructures. Instead, we observe a very weak first-order transition for the slowest cooling rate $(1.37 \mathrm{~K} / \mathrm{ps})$, where the discontinuity is too small to measure in our simulations. At intermediate cooling rates, this phase transition becomes quasi-continuous (6.7$3.35 \mathrm{~K} / \mathrm{ps})$ and is clearly continuous at faster cooling rates $(67 \mathrm{~K} / \mathrm{ps})$. The implications of the 
kinetics and nature of phase transition on the structural evolution and distribution of various defect states are rather interesting and will be discussed in the next sub-section.

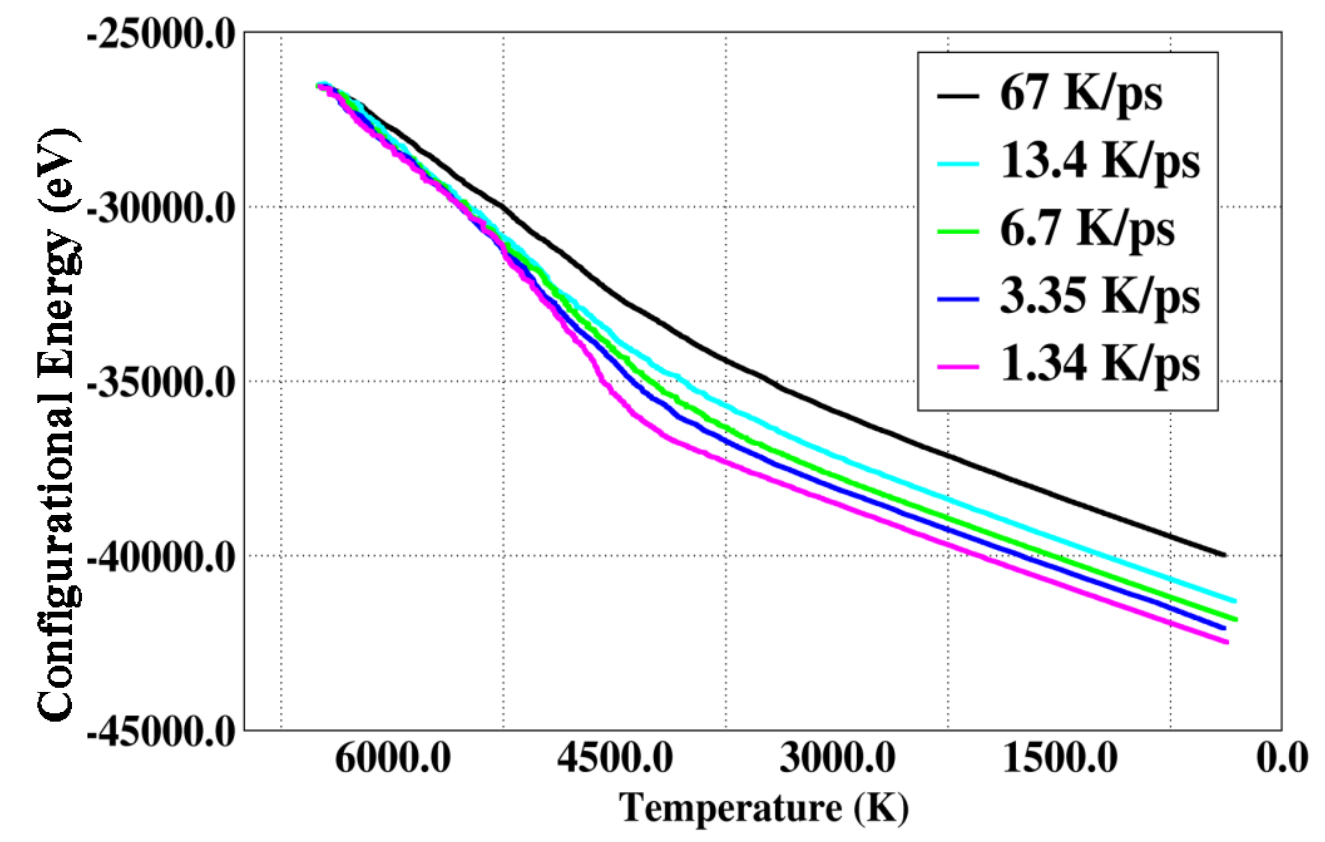

Figure 2. Variation of configurational energy of completely dehydrogenated polyethylene system during the various cooling runs (a) $67 \mathrm{~K} / \mathrm{ps}$ (b) $13.4 \mathrm{~K} / \mathrm{ps}$ (c) $6.7 \mathrm{~K} / \mathrm{ps}$ (d) $3.35 \mathrm{~K} / \mathrm{ps}$ (e) $1.34 \mathrm{~K} / \mathrm{ps}$.

\subsubsection{Reaction mechanism for formation of carbon nanostructures from amorphous melts}

Before investigating the influence of the cooling kinetics on the structure and the defect evolution, it is important to understand the various reaction mechanisms that lead to nucleation of the carbon nanostructures. A radical chain mechanism is the more widely accepted reaction pathway to explain the pyrolysis of PE. The radical chain model initiates with a random scission of PE chains that leads to formation of primary radicals. The various steps identified in conventional pyrolysis consist of: (1) scission in $\beta$ of these radicals, (2) intra-molecular hydrogen transfer, followed by scission, and (3) intermolecular hydrogen transfer [50-54]. The termination followed by pr opagation by a recombination of radicals forms shorter hydrocarbons. Potsuma reports a comprehensive study on the mechanisms associated with 
breakdown of PE in open systems [52, 53]. These mechanisms include breakdown of C-C bonds in the backbone at random locations leading to numerous short hydrocarbons with varying number of $\mathrm{C}$ atoms, formation of volatile products via scission/backbiting near the ends of the PE, regeneration of monomer via unzipping process, and increase in polymer branching. However, it should be noted that earlier works on pyrolysis of PE were primarily focused on ope $n$ systems, which leads to the degradation of PE into short hydrocarbons/volatiles and/or the monomer ethylene.

In the current study, our efforts are focused on a more closed system, in which, pressures ( $\sim 1000 \mathrm{psi})$ are self-generated owing to the formation of the gaseous products (e.g, volatile hydrocarbons, hydrogen etc.). In addition, the exothermic nature of these reactions increase the temperature to $>=\sim 1073 \mathrm{~K}\left(>=800{ }^{\circ} \mathrm{C}\right)$. Under these conditions of pressure and temperature, the carbonaceous mass obtained after pyrolysis coalesce, and graphitizes leading to carbon nanostructures, e.g., carbon onions, spheres etc. Such nanostructures are novel and could not be formed in the earlier works of PE degradation, since those systems were open, wherein the gaseous products formed during degradation are free to escape.

It is extremely difficult (nearly impossible) with the current state-of-the-art characterization techniques to explore the atomic level mechanism of PE degradation during high temperature pyrolysis reaction. In addition, since we have a closed system, it is very difficult to experimentally predict the reaction pathways that lead to formation of CS only based on the final product distribution. Our previous simulations suggest that the initial stage in the degradation of PE chain is primarily via random scission of the backbone $\mathrm{C}-\mathrm{C}$ bond in $\mathrm{PE}$ [23]. This leads to formation of shorter hydrocarbons out of which a fraction of $\mathrm{C}-\mathrm{H}$ bonds dissociate. This is not unexpected as the $\mathrm{C}-\mathrm{C}$ bonds are significantly weaker ( $\sigma$ bond 
dissociation energy $=\sim 347 \mathrm{~kJ} / \mathrm{mol}$ ) when compared to the $\mathrm{C}-\mathrm{H}$ bonds (bond dissociation energy $=\sim 414 \mathrm{~kJ} / \mathrm{mol})[55,56]$. Similar bond cleavages has been recognized in previous experimental and theoretical studies [54].

Based on t hese thermodynamic considerations, we had reported previously a mechanism for the formation of carbon nanostructures:

1) $\mathrm{PE}$ decomposes via a series of decomposition reactions into shorter chain hydrocarbon fragments.

$$
C_{n} H_{n} \Leftrightarrow C_{n-m} H_{n-m}+C_{m} H_{m} \quad(\mathrm{~m}<\mathrm{n})
$$

2) Breakage of the $\mathrm{C}-\mathrm{H}$ bond in short chain hydrocarbons to release hydrogen.

$$
C_{m} H_{m} \Leftrightarrow C_{m} H_{m-p}+p H \quad(\mathrm{p}<\mathrm{n})
$$

3) Dissociated carbon polymerizes to form carbon chains.

$$
n C \Leftrightarrow C_{n}
$$

4) Free hydrogen either combine to form hydrogen gas molecules or recombine with dissociated carbon to form short chain hydrocarbons.

$$
H+H \Leftrightarrow H_{2}
$$

These intermediate reactions are coupled and generally compete amongst each other.

We note in our simulations that the initial dissociation process at $7000 \mathrm{~K}$ is quite rapid: $\sim 80 \%$ of hydrogen atoms in the PE chain dissociate within the first 100 ps. The evolution and phase separation of this hydrogen is simultaneously accompanied by polymerization of the carbon atoms. During the polymerization process, the carbon atoms form stronger $\sigma$ double or triple bonds among themselves. Once PE is thermally degraded into smaller linear hydrocarbons, the mechanisms associated with the cyclization/formation of graphite like structures closely resemble those involved in soot formation [57-60]. For instance, in the soot 
formation literature, it is well accepted that the polymerization of short hydrocarbon chains proceeds via series of repetitive steps including hydrogen abstraction followed by addition of acetylene.

In the present study, we observe similar reaction steps in our MD simulations. It is important to note that the reactions described above are reversible, and that the conditions of temperature/pressure play a $\mathrm{k}$ ey role in the progress of the polymerization reaction. Furthermore, as reported by Frenklach et al., the cyclization of short linear hydrocarbon chains is associated with energy barriers; these barriers can be surmounted by c arefully chosen reaction conditions (temperature, pressure) [57, 58]. The cyclization can lead to formation of rings of different members $(5,6,7$ etc.) depending on whose formation energy barriers can be surmounted for certain external conditions. Effects of temperature, fuel identity, and additives, etc., upon soot formation have been studied extensively in experiments. In the case of soot formation, the end product is graphitized nanostructure at high temperatures whereas they are amorphous at low temperatures. Moreover, once carbonization occurs, the nanostructure of the soot particle becomes basically locked in at ordinary flame temperatures. We note that these are very different from the compact hard spheres that form in the present study owing to the different temperature-pressure conditions and the resulting structure of the end-product depends strongly on the kinetics of the cooling process.

In the present work, we show that cooling rate provides an effective route to tune the reactions pathways and consequently, exercise control over the nucleation and growth processes during crystallization of carbon melts. We employ different cooling rates, which take advantage of the coupling between the reversibility of the reactions, and the presence of energy barriers to control the formation and coordination of rings (e.g., pentagon vs hexagon). The 
cooling rate offers such tuning, since it decides the amount of time the system spends at high temperatures, and in turn, impacts the reaction kinetics. By carefully choosing the cooling rate, we can specifically control the formation of one type of rings versus the other (i.e. tune the reaction pathway). This, in turn, enables synthesis of tailored carbon nanostructures, in which the defect states and their distribution can be precisely controlled to obtain prescribed physical properties.

\subsubsection{Effect of cooling rate on the structural evolution of the carbon nanostructures}

To quantitatively assess the impact of kinetics on the extent of structural order, we evaluate the radial distribution functions (RDF) in the crystallized nanostructures at $300 \mathrm{~K}$ obtained from our MD simulations at various cooling rates [Figure 3 (a) and (c)]. In addition to compare the nanoscale structural and morphological differences between experimentally produced and simulated CS, we have calculated the experimental neutron scattering pair distribution function (PDF) analysis [Figure 3 (b)]. In the RDF for the C-C pairs [Figure 3 (a)], peaks arising from the nearest and next-nearest neighbors occur at similar separation distances

of $\sim 1.45 \AA$ and $\sim 2.45 \AA$ respectively, regardless of the cooling rate employed. Thus, shortrange order in the obtained carbon nanostructures is not affected by the kinetics of the crystallization. The long-range order, however, exhibits a strong dependence on the cooling rates. At slow cooling rates, the C-C RDF shows well-defined higher order peaks while they are absent at fast rates [Figure 3; Ref. 61]. 

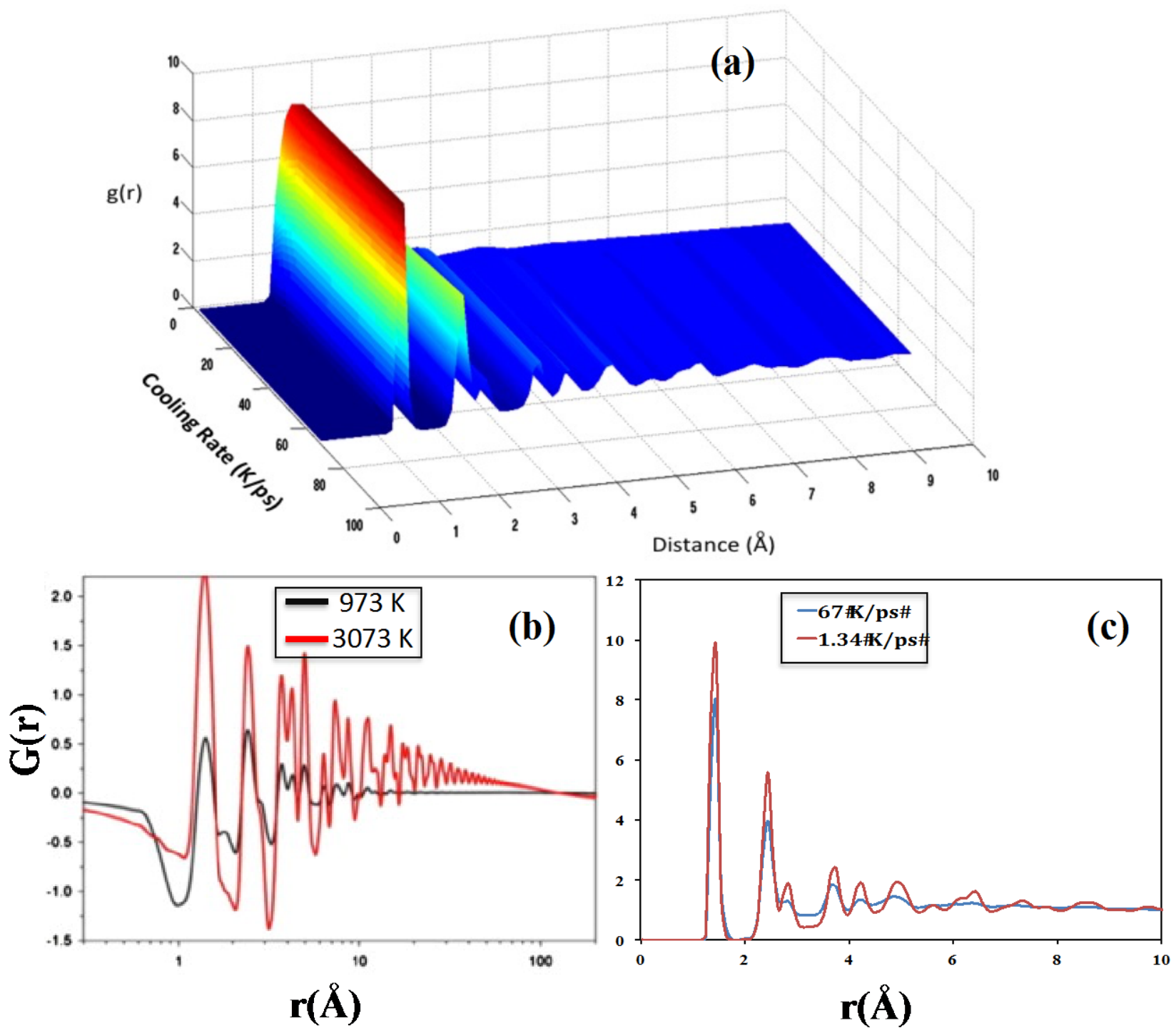

Figure 3. (a) Radial distribution function (RDF), obtained from MD simulations, of the crystallized carbon nanostructures at $300 \mathrm{~K}$ as a function of the cooling rate. It can be seen that the long-range ordering is dictated by the cooling rate. (b) Experimental neutron scattering pair distribution function (PDF) for carbon microsphere cooled from $\sim 973 \mathrm{~K}\left(700{ }^{\circ} \mathrm{C}\right)$ and $\sim 3073 \mathrm{~K}\left(2800{ }^{\circ} \mathrm{C}\right)$ to room temperature. Long range ordering can be observed for structures cooled from $\sim 3073 \mathrm{~K}\left(2800{ }^{\circ} \mathrm{C}\right)$ to room temperature (c) RDF obtained from MD simulations for nanoscale amorphous carbon nanostructures with cooling rates of $67 \mathrm{~K} / \mathrm{ps}$ and $1.34 \mathrm{~K} / \mathrm{ps}$. Long range ordering can be observed for nanostructures with lower cooling rates, namely, $1.34 \mathrm{~K} / \mathrm{ps}$.

In addition, a prominent shoulder appears at $\sim 2.85 \AA$ in the $\mathrm{C}-\mathrm{C}$ RDF at slower cooling rates; in fact its intensity increases progressively as co oling rate is reduced. This peak corresponds to the intra-hexagon spacing, which suggests the formation of a more compact, 
homogeneous, and less defective structure with graphitic order at slow rates (as seen in the snapshots shown in Figure 4).

The PDF of experimentally produced CS cooled from $3073 \mathrm{~K}\left(2800{ }^{\circ} \mathrm{C}\right)$ to room temperature show strong evidence for graphitic-like character and increased order at longrange (Figure 3 (b)). This observation resembles the carbon-carbon PDF calculated for the CS formed at lower cooling rates $(1.34 \mathrm{~K} / \mathrm{ps})$ in our MD simulations (Figure 3 (c)). At lower cooling rates the C-C RDF shows well-defined higher order peaks and increased graphitic order. Note, at lower cooling rates, the system resides for a longer time at higher temperatures that is equivalent to natural cooling from $3073 \mathrm{~K}\left(2800{ }^{\circ} \mathrm{C}\right)$ to room temperature. On the other hand, the experimental PDF for CS cooled from $973 \mathrm{~K}\left(700^{\circ} \mathrm{C}\right)$ to room temperature shows a distinct negative peak at $\neg 1.0 \AA$ (Figure 3 (b)). The negative scattering length of hydrogen indicates $\mathrm{C}-\mathrm{H}$ bonding and confirms that hydrogen is present in these $\mathrm{CS}$ at an approximate overall ratio of $\mathrm{C}-\mathrm{H}$ to $\mathrm{C}-\mathrm{C}$ bonds of -0.15 . In addition, experimental $\mathrm{PDF}$ also show the decrease in long-range and graphitic order in these CS. Similar decrease in long-range and graphitic order can be observed in the simulated PDF for the CS formed at higher cooling rates, namely, $67 \mathrm{~K} / \mathrm{ps}$ (Figure 3 (c)).

Thus, in our simulations, at $300 \mathrm{~K}$ all the obtained structures exhibit evidence of crystallization of carbon atoms irrespective of the cooling rates; however, the extent of graphitic order progressively increases with decrease in cooling rates [Figure 4]. In particular, the carbon structures produced at lower cooling rates $(1.34 \mathrm{~K} / \mathrm{ps})$ closely resemble the concentric nanometric texture [41]. It could be the result of cooling of carbon nanostructures from temperatures as high as $\sim 7000 \mathrm{~K}$ to room temperature, at lower cooling rates, where we observe the solidification of liquid carbon to form graphene layers parallel to the surface of the 
spheres. On the other hand, in the case of higher cooling rates of $67 \mathrm{~K} / \mathrm{ps}$ the CS consisting of random or glass-like carbon are formed.

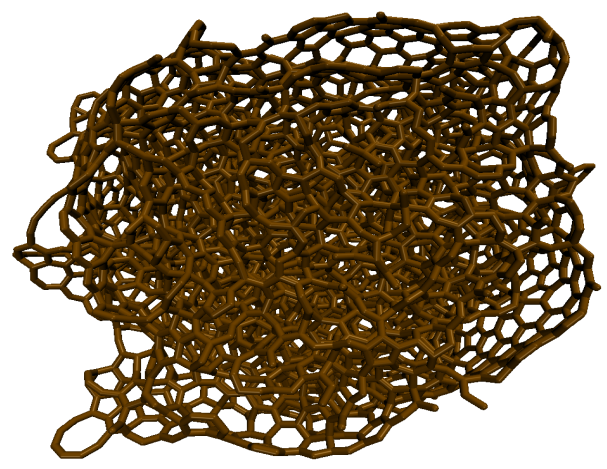

(a)

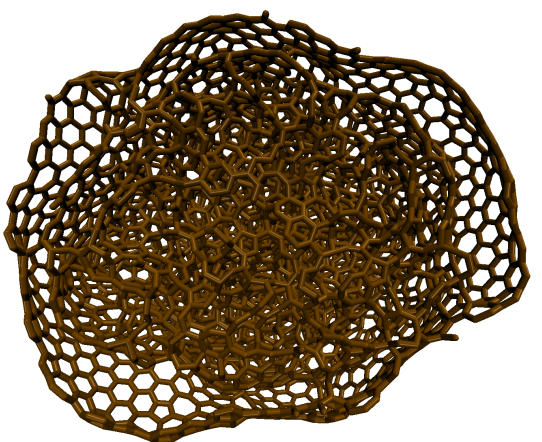

(b)

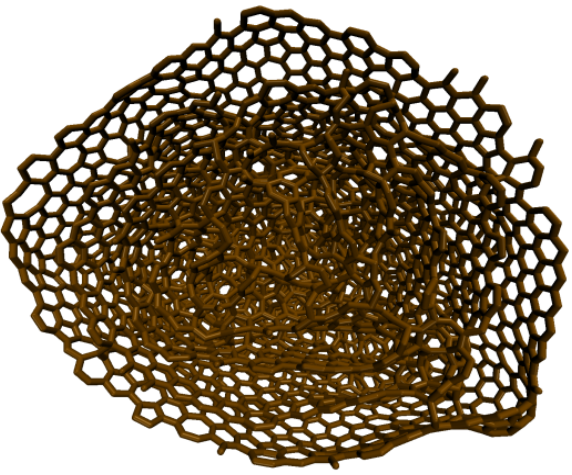

(c)

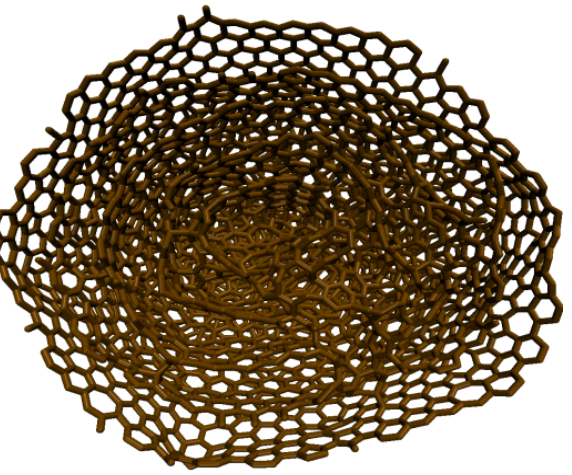

(d)

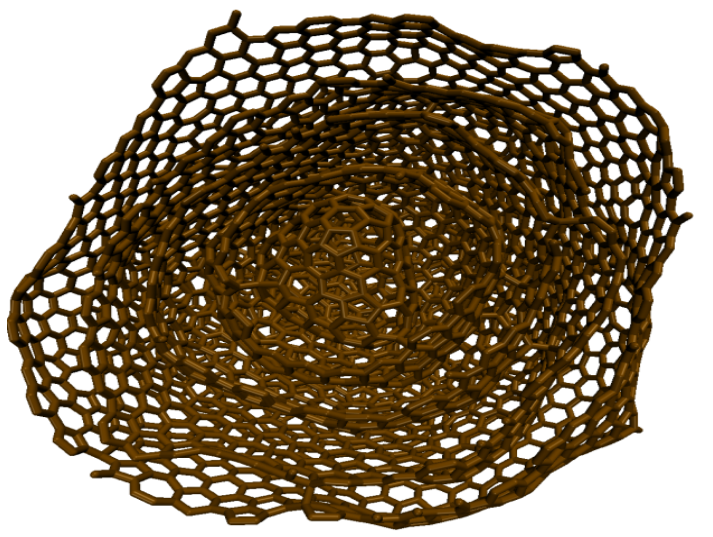

(e)

Figure 4. Snapshots showing the effect of various cooling rates on the structure of the carbon nanostructures. The structures shown represent the cooled configurations obtained at $300 \mathrm{~K}$ for (a) $67 \mathrm{~K} / \mathrm{ps}$ (b) $13.4 \mathrm{~K} / \mathrm{ps}$ (c) $6.7 \mathrm{~K} / \mathrm{ps}$ (d) $3.35 \mathrm{~K} / \mathrm{ps}$ (e) $1.34 \mathrm{~K} / \mathrm{ps}$ cooling rates. Note, the structures depicted here are typically $4-5 \mathrm{~nm}$ in diameter. 
To study the effect of cooling rates on the formation of defect sites, we identify the nature and fraction of $n$-membered rings $(n=5-12)$ in the carbon nanostructure. There are a myriad of different defects (shown in the schematic in Figure 5) that can arise by breaking the symmetry of the honeycomb lattice via various bond rotations. For example, SW defect can be formed by transforming four hexagons into two pentagons and two heptagons (SW) (55-77) defect shown in Figure 5(b) by rotating one of the C-C bonds by $90^{\circ}$. It should be noted that the formation of the density of double vacancies (DV) or SW defects is usually small under natural cooling or low temperature formation processes due to the high activation barrier of several $\mathrm{eV}(\sim 5 \mathrm{eV})$ required for the bond rotation. Thus, the high formation energy of the SW defect indicates a negligible equilibrium concentration under typical experimental synthesis conditions of $\sim 1273 \mathrm{~K}\left(1000{ }^{\circ} \mathrm{C}\right)$ or less. The reverse transformation to a hexagon also has an energy barrier of $\sim 5 \mathrm{eV}$. Note that the high reverse energy barrier ensures that once the defect is formed under non-equilibrium conditions, it would remain stable at room temperatures. As mentioned earlier, controlled formation of the various defects is important in various applications from catalysis to electronics but achieving a precise control on defect distribution has remained a major challenge owing to the defect energetics. While the schematic shown in Figure 5 depicts a typical DV and various SW defects, it is easy to quantify the defects by following the temporal evolution of the various $n$-membered rings. To examine the effect of cooling rate on formation of unique n-membered ring sites of various carbon nanostructures, we utilize the algorithm developed by Yuan et al. to quantify these random nano-networks based on carbon ring sizes [62]. The relative weights in the ring statistics provide a quantitative estimate of the population distribution of the various defective sites. Figure 6 (a) - (f) show the ring probability of the formation of 6-12 membered rings during different cooling runs. 


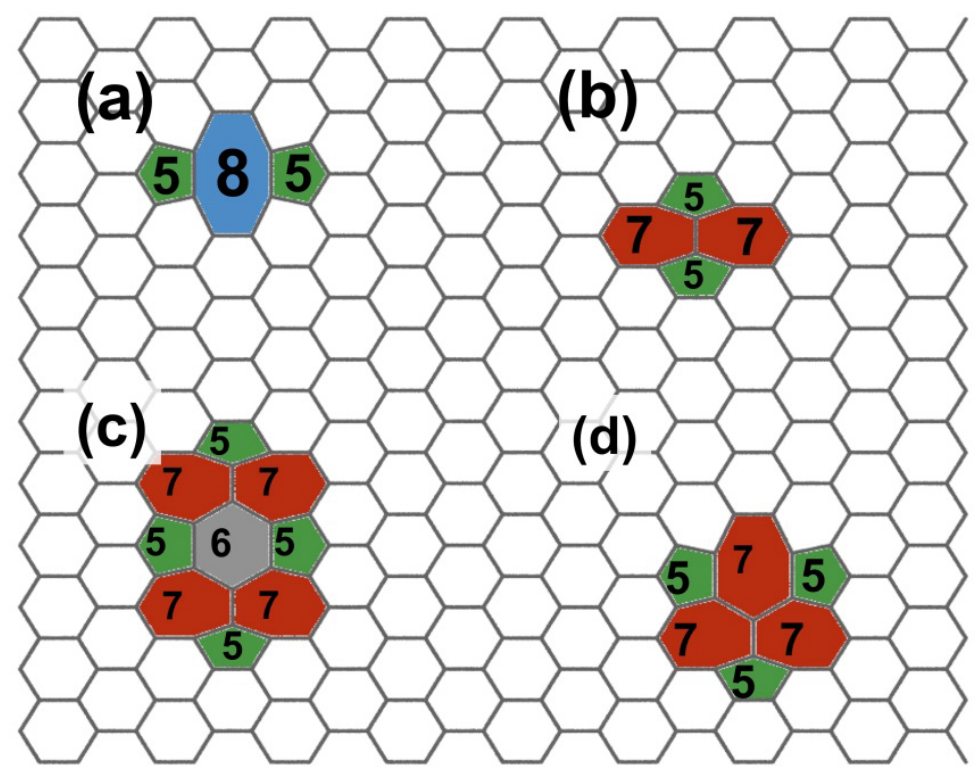

Figure 5. Schematic of the ring distribution corresponding to various commonly found defects in graphene (a) Double vacancy (b)-(d) various representation of the Stone-Wales (SW) defect. Note that the double vacancy consists of an eight membered ring and two adjacent five membered rings whereas (b)-(d) comprises of various bond rotations of seven-five membered rings.

In the case of faster cooling rates, namely at $67 \mathrm{~K} / \mathrm{ps}$, we do not find any significant difference in the distribution of various $n$-membered rings. This is not surprising, as under the highly non-equilibrium conditions, one would expect the nucleation and growth of graphitic rings to compete with each other over an extended temperature range from 5000-3000 K. Moreover, the shorter relaxation times as compared to other cooling rates considered in the present study coupled with the high defect formation energies ensures that the defects remain stable after their initial formation and during the subsequent cooling stage. This is in accordance with the experimental observation shown in Figure 1 as well as the continuous transformation observed in the configurational energy curve shown in Figure 2.

Figure 6 suggests that at cooling rates $\leq 13.4 \mathrm{~K} / \mathrm{ps}$, there is an initial nucleation stage followed by a slower growth. This occurrence of nucleation and growth over distinct time 
domain is further evidenced by sharp change in the slope of configurational energy plotted as a function of cooling rate (similar to a first-order transition), particularly at rates $\leq 3.35 \mathrm{~K} / \mathrm{ps}$. At $\mathrm{T}>4500 \mathrm{~K}$, carbon atoms are in liquid state leading to formation of more defective sites $(>7$ membered rings) similar to amorphous structures. As the temperature drops, the fraction of these defective sites decreases significantly. Nucleation occurs at temperature $\sim 4500 \mathrm{~K}$ with growth occurring at lower temperatures. During this growth phase, the number of more stable 6-membered rings increases significantly with near perfect graphitic arrangement at rates $\leq 3.35$ $\mathrm{K} / \mathrm{ps}$. In the intermediate cooling rate regime $(3.35-13.4 \mathrm{~K} / \mathrm{ps})$, we observe an increase in 5and 7-membered rings.
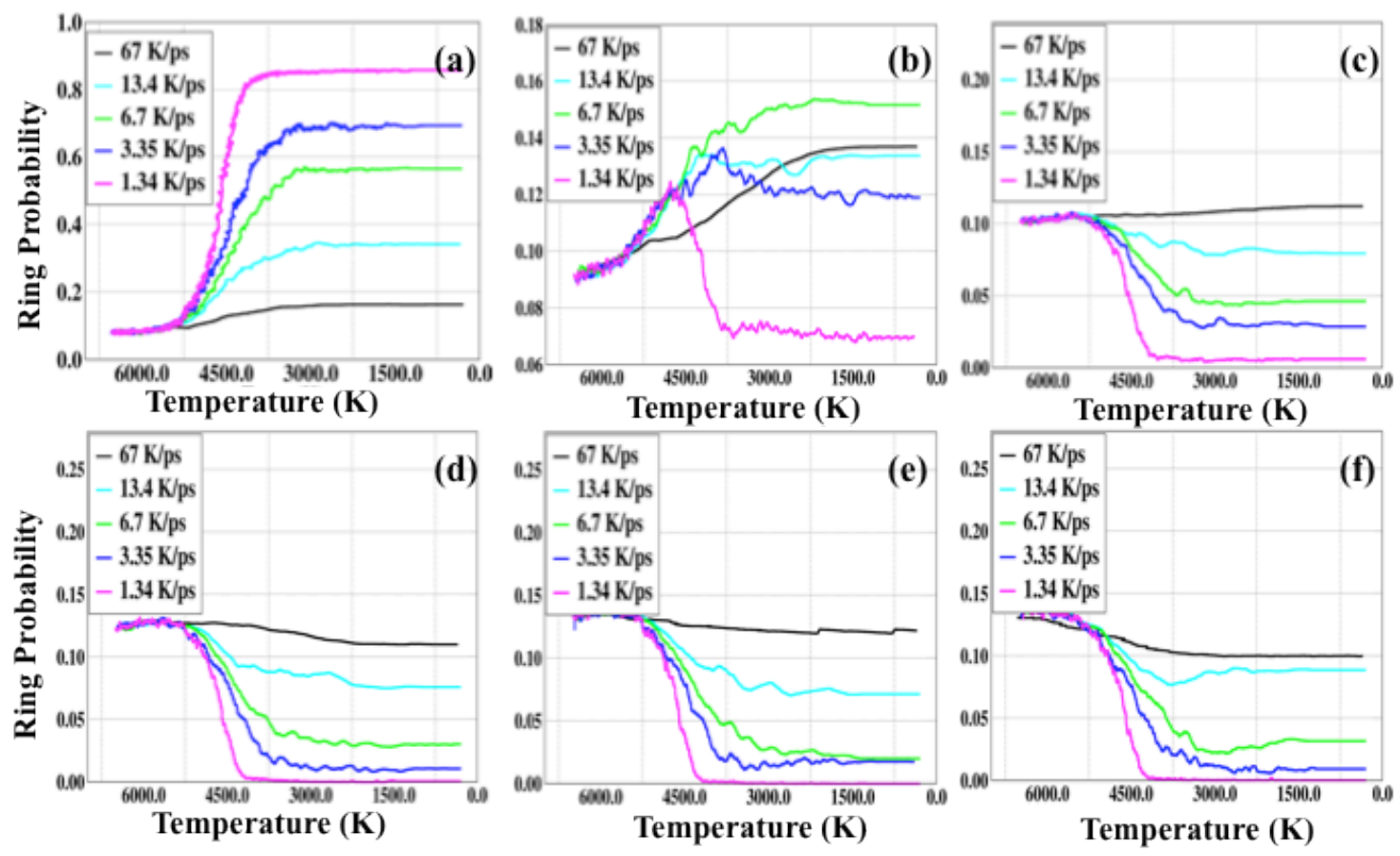

Figure 6. Defect evolution characterized graphitic order during the various cooling runs for $0.1 \%$ hydrogen content system (a) 6 Membered Ring (b) 7 Membered Ring (c) 8 Membered Ring (d) 10 Membered Ring (e) 11 Membered Ring (f) 12 Membered Ring.

Figure 7 (a) - (e) shows the snapshots revealing the cross-section of the final configuration of carbon nanostructures at $300 \mathrm{~K}$ for different cooling rates. The defects 
represented by rings are colored by their sizes. Carbon rings with 5-, 6-, 7-, $\geq 8$-membered rings are shown in green, gray, red, and blue, respectively. From Figure 7 it can be clearly seen that the fraction of $\geq 8$-membered rings (blue rings) progressively reduces as we move from Figure 7 (a) to (e) i.e. from non-equilibrium or faster cooling rate towards more equilibrium or slower cooling rate. Concurrently, we find that the fraction of 6-membered rings (gray rings) increases with decrease in the cooling rate. Specifically, when we move from Figure 7 (a) to (e), the fraction of defects decreases. In addition, we also find presence of a significant fraction of the 5-membered rings in all the nanostructures shown in Figure 7 (a) to (e). Five-membered rings embedded in a hexagonal lattice, similar to those observed in fullerenes, impart geodesic curvature [63]. In the case of 7-membered rings, we find that their fraction reduces as we go from faster cooling rates to slower cooling rates. The reduced 5- and 7- membered rings imply lesser fraction of SW defects at slower cooling rates.

(a)

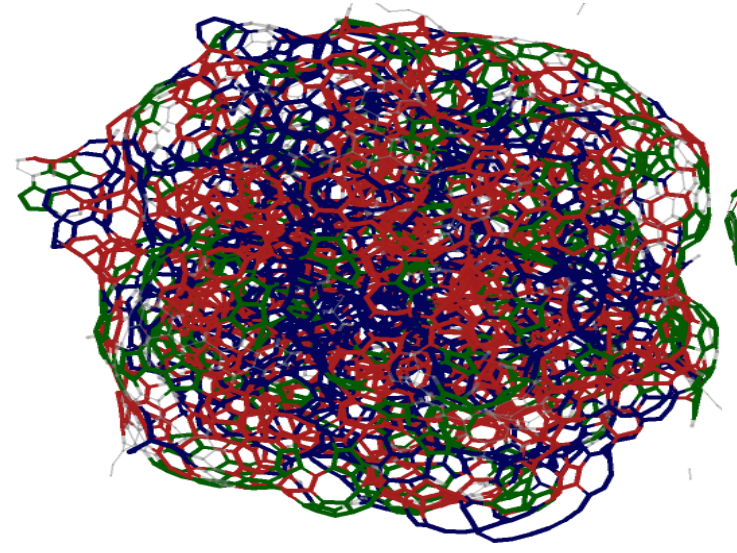

(b)

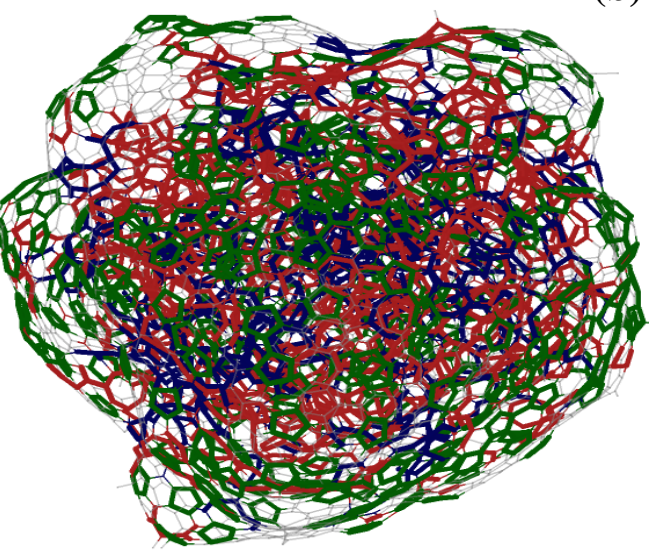


(c)

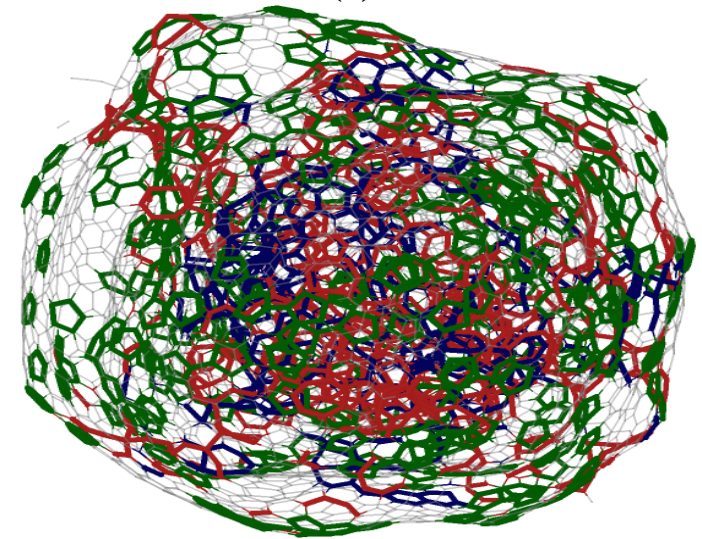

(e)

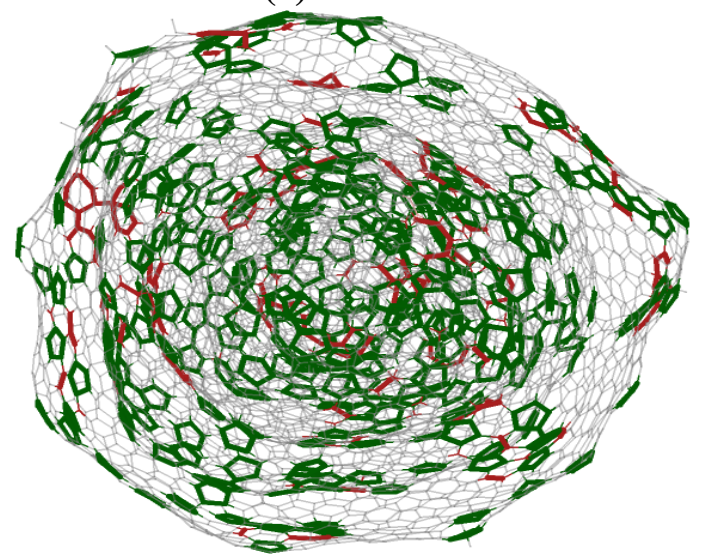

(d)

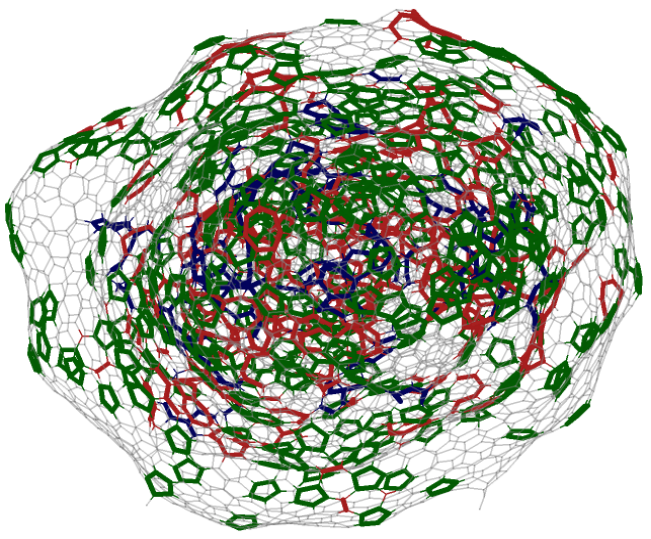

Figure 7.Morphology of the carbon nanostructures at $300 \mathrm{~K}$ obtained via cooling at various rates (a) $67 \mathrm{~K} / \mathrm{ps}$ (b) $13.4 \mathrm{~K} / \mathrm{ps}$ (c) $6.7 \mathrm{~K} / \mathrm{ps}$ (d) $3.35 \mathrm{~K} / \mathrm{ps}$ (e) $1.34 \mathrm{~K} / \mathrm{ps}$. Crosssection of the final configurations are shown and the topological C-rings of different sizes are highlighted. The defects represented by rings are colored by their sizes: 5-, 6-, 7-, , 8membered rings are shown in green, gray, red, and blue, respectively.

3.2.5 Graphitic order in carbon nanostructures at room temperature in the cooled configuration

We further evaluate the defect distribution in the final cooled configuration. Figure 8 shows the ring statistics calculated at the end of cooling run at $300 \mathrm{~K}$ for various cooling rates. Due to lack of both angular and torsional strain 6-membered rings have least strain; hence in these carbon nanostructures we find that the formation of 6-membered rings is most favored over others [See Figure 8]. However, we do obs erve that the distribution of defect sites is strongly dependent on the cooling rates. In the case of $1.34 \mathrm{~K} / \mathrm{ps}$ cooling rate, the ring statistics reveals a narrow distribution with highest fraction of 6-membered and lowest fraction of defect 
sites $(n \neq 6)$. With the increase in the cooling rate from $1.34 \mathrm{~K} / \mathrm{ps}$ to $67 \mathrm{~K} / \mathrm{ps}$ we find that the fraction of 6-membered rings decreases and formation of defective carbon sites consisting of $\mathrm{n}$ $>6$-membered carbon rings increases. The increase in defect sites observed at higher cooling rates could give rise to the quench effects. In other words, the loss in mobility of carbon atoms during the growth phase before they diffuse/rearrange themselves to achieve the most favored 6-membered ring structures. Thus, the fraction of defective carbon sites (number of $\mathrm{n}>6$ membered rings) was found to be substantial for configurations with high cooling rates i.e. those that were cooled at $67 \mathrm{~K} / \mathrm{ps}$. On the other hand, however, for slower cooling rates carbon atom has enough time to diffuse and rearrange itself to form strain free 6-membered rings.

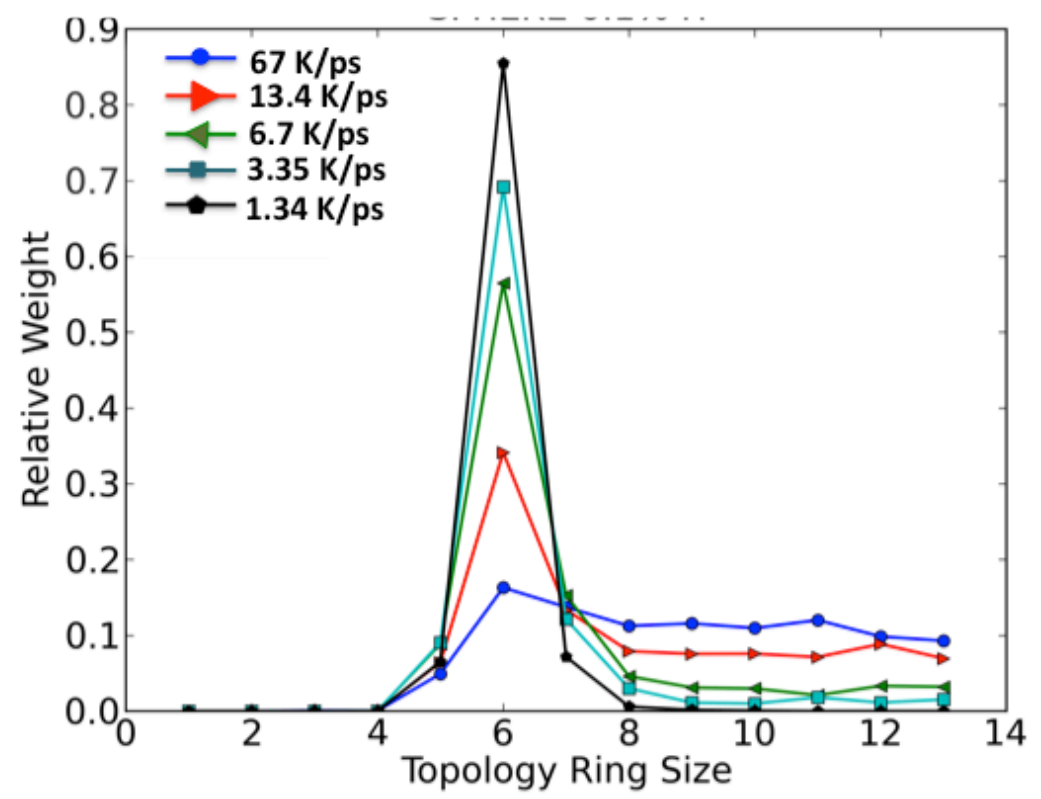

Figure 8.Ring statistics for crystallized nanocarbon at $300 \mathrm{~K}$. A cutoff radius of $1.95 \AA$ is used to define inter-atomic neighbors distance. The completely dehydrogenated PE systems were cooled to $300 \mathrm{~K}$ with various different cooling rates.

\section{(3.2.6) Effect of residual hydrogen content on the dynamics of crystallization}

We further study the effect of the residual hydrogen content on the kinetics of crystallization of amorphous carbon melts with different hydrogen content. During the heating 
process at $7000 \mathrm{~K}$, after every $1 \mathrm{ps}$, we examine the simulation cell for the presence of free hydrogen or a dimer of hydrogen based on the bond cut-off defined by the REBO potential $(\sim 1.1 \AA)$ and remove them from the simulation cell. This leads us to systems with varying hydrogen content. As a representative test case, systems with varying hydrogen content, namely, $0.1,0.5,10$, a nd $20 \%$ were cooled to room temperature with two different cooling rates namely, $67 \mathrm{~K} / \mathrm{ps}$ and $1.34 \mathrm{~K} / \mathrm{ps}$. Figure 9 (a) and (b) shows the variation in the configurational energy of the system as a function of hydrogen content and cooling rates. It can be clearly seen from both Figure 9 (a) and (b) that the configurational energy decreases with decrease in temperature. The temperature range over which the liquid carbon transforms into solid phase (nucleation of graphitic structures) occurs at $\sim 4000-5000 \mathrm{~K}$ and strongly depends on the hydrogen content and cooling rate.

At higher cooling rates of $67 \mathrm{~K} / \mathrm{ps}$, as shown in Figure 9(a), the slope of the configuration energy versus temperature plot remains nearly constant for all values of residual hydrogen. This suggests that at higher cooling rates the liquid-solid transition is quasicontinuous, regardless of the presence/absence of residual hydrogen. This could be attributed to a uniform distribution of nucleation sites, and reduced mobility of carbon atoms due to quenching-like effect.

Figure 9 (b) shows the configurationally energy plots for the systems cooled at the slowest rates $(1.34 \mathrm{~K} / \mathrm{ps})$ at varying levels of residual hydrogen content. In these cases, the plots exhibit a sharp change in slope at temperature range $\sim 4000-5000 \mathrm{~K}$, in contrast to their counterparts at fast rates [Figures $9(\mathrm{a}, \mathrm{b})]$. This temperature range (over which slope changes) marks the liquid-solid transition regime via a nucleation-growth mechanism. Higher temperature $(\sim 5000 \mathrm{~K})$ corresponds to formation of nuclei, while the low temperature ( $\sim 4000$ 
K) marks the onset of growth phase, which eventually leads to the formation of ordered carbon nanostructures. A careful inspection of Figure 9(b) reveals that as the residual hydrogen content increases, both these temperatures (corresponding to nucleation and growth events) shift towards lower values. For example, at $20 \%$ hydrogen content, nucleation occurs at $\sim 4000$ $\mathrm{K}$ as compared to $\sim 5000 \mathrm{~K}$ for $0.1 \%$ residual hydrogen. Thus, the residual hydrogen content controls the onset temperature for nucleation and growth; higher hydrogen content leads to nucleation and growth at lower temperatures.
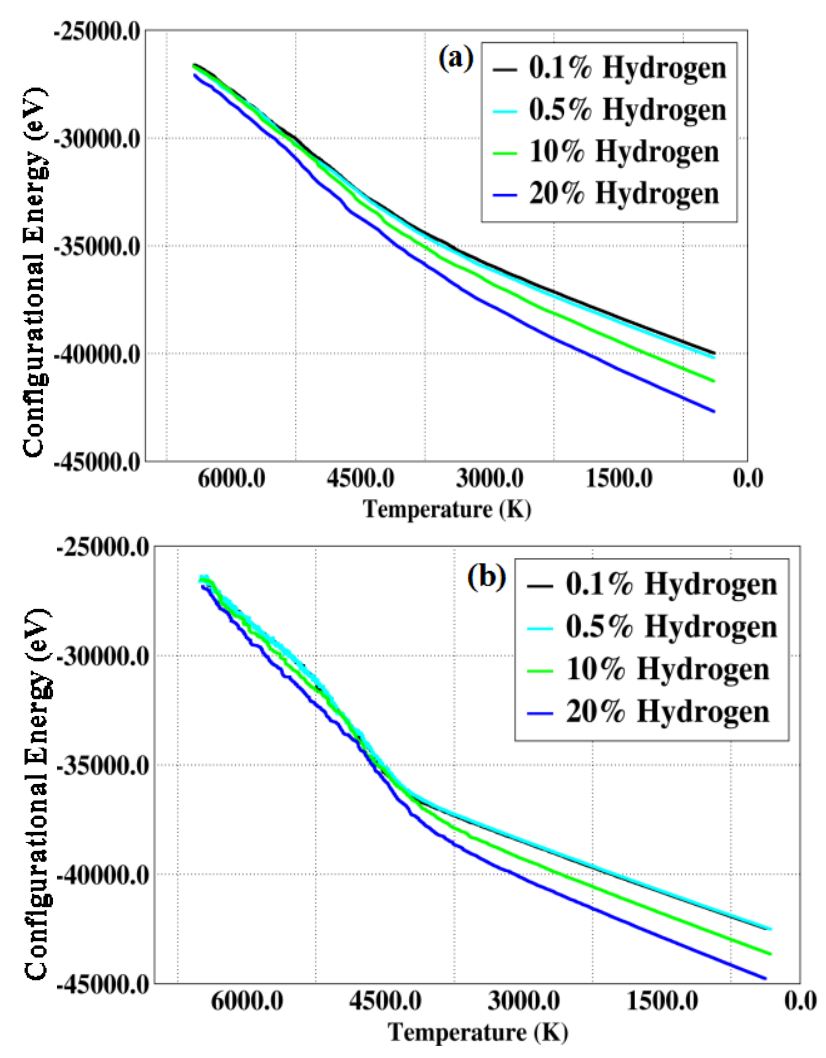

Figure 9. Effect of residual hydrogen content on the dynamics of crystallization of carbon nanostructures (a) $67 \mathrm{~K} / \mathrm{ps}$ (b) $1.34 \mathrm{~K} / \mathrm{ps}$.

\section{(3.2.7) Effect of residual hydrogen content on the structure of carbon nanostructures}

Although residual hydrogen content governs the temperature at which nucleation and growth events commence, it does not impact the spatial order of carbon atoms in the final nanostructure. Figure 10 (a) and (b) shows the RDF for carbon-carbon atom pair of structures 
for various residual hydrogen content at two different cooling rates $67 \mathrm{~K} / \mathrm{ps}$ and $1.34 \mathrm{~K} / \mathrm{ps}$, respectively. We note that these RDFs were evaluated for the final carbon nanostructure obtained at $300 \mathrm{~K}$.

As shown in Figure 10 (a), in the ultra-fast cooling regime $(67 \mathrm{~K} / \mathrm{ps})$, the obtained carbon nanostructures are highly disordered, irrespective of residual hydrogen content. At such fast cooling rates, the long-range order of carbon nanostructures (similar to that seen in Figure 3 ) is lost due to quenching effects [22]. In terms of short-range order, the positions of first $(\sim 1.45 \AA)$ and second neighbor $(\sim 2.45 \AA)$ peaks in the C-C RDFs remains invariant with respect to residual hydrogen. The intensity of these peaks, albeit, increase at lower residual hydrogen levels.

Similarly, at slower cooling rates $(\sim 1.34 \mathrm{~K} / \mathrm{ps})$ ordered and homogeneous structures are obtained regardless of the hydrogen content [Figure 10(b)]. Here, in addition to short-range (i.e., first and second neighbor) peaks, a prominent shoulder is observed at $\sim 2.85 \AA$ in the C-C RDF, similar to Figure 3. As aforementioned, this peak arises from carbon pairs within a hexagon, indicating the formation of a structure predominantly consisting of 6-membered rings. Here again, the intensity of these peaks increases with reduction in hydrogen content without influencing their positions. 


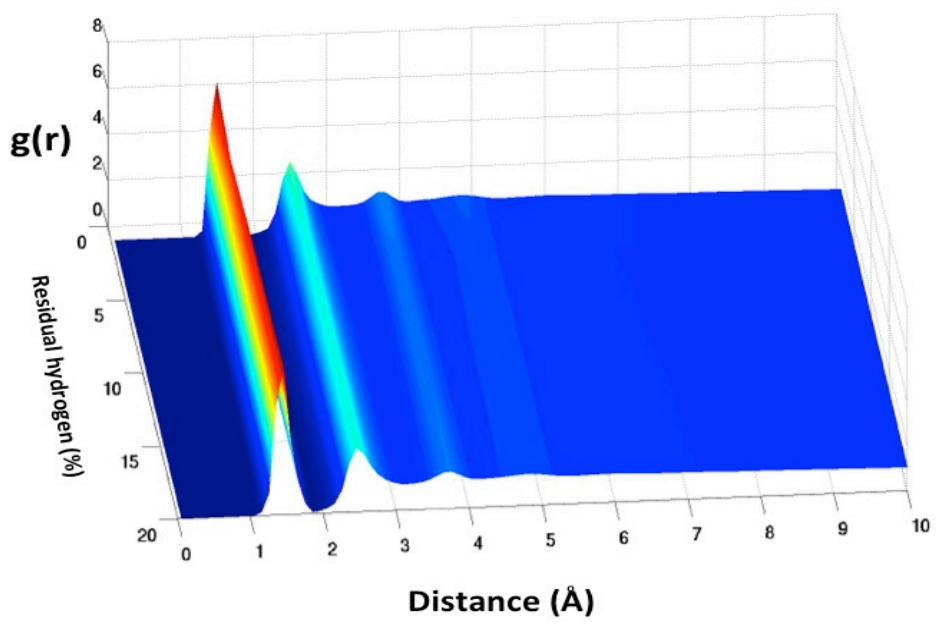

(a)

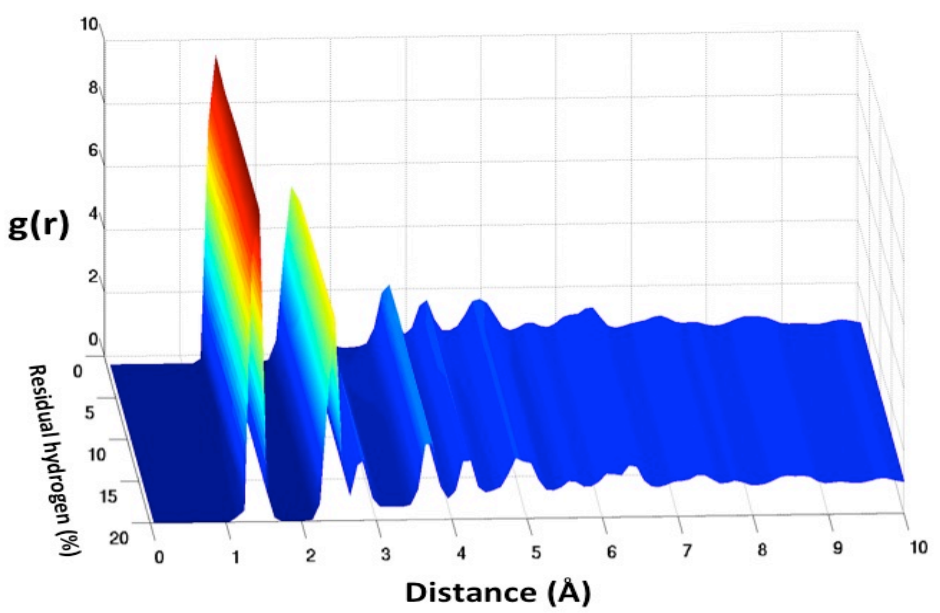

(b)

Figure 10. Radial distribution function (RDF) of carbon-carbon atom pairs ( $g(r) C-C)$ for systems with various residual hydrogen contents at $300 \mathrm{~K}$. The RDF is calculated for the crystallized configurations at $300 \mathrm{~K}$. Two different cooling rates are shown (a) $67 \mathrm{~K} / \mathrm{ps}$ (b) $1.34 \mathrm{~K} / \mathrm{ps}$.

(3.2.8) Combined effect of cooling rates and residual hydrogen content on the graphitic order of carbon nanostructures

We now evaluate the effect of the cooling rates and residual hydrogen content on the structural evolution of carbon nanostructures. Figure 11 (a) and (b) show the ring statistics for systems with various residual hydrogen contents for two representative cooling rates i.e. 67 $\mathrm{K} / \mathrm{ps}$ and $1.34 \mathrm{~K} / \mathrm{ps}$, respectively. The data are collected at the end of cooling process at $300 \mathrm{~K}$. 
We find that the graphitic order of carbon nanostructure is strongly dependent on both the cooling rate and hydrogen content. Figure 11 (a) shows that at a given fast cooling rate (67 $\mathrm{K} / \mathrm{ps}$ ), the overall fraction of carbon rings reduces with an increase in the hydrogen content. The fraction of 6-membered rings is marginally higher when compared to the other carbon rings. At higher hydrogen content, carbon atoms react more readily with the hydrogen atoms stabilizing the defects and forming hydrocarbon side products. Indeed, we find a broader distribution of carbon rings ( $\mathrm{n}=5$ to 13 ) in Figure 11(a) at high residual hydrogen content. In the case of slower cooling rates, namely at $1.34 \mathrm{~K} / \mathrm{ps}$, the ring statistics reveals that the effect of residual hydrogen is less pronounced [Figure 11(b)]. Owing to longer relaxation times at slower cooling rates, the structures formed are less defective as shown by a sharp distribution around 6-membered rings. Their fraction decreases only slightly with an increase in hydrogen content from 0.1 to $20 \%$.

During the cooling phase, the presence of higher $\mathrm{H}$ content can either lead to reaction of highly reactive carbon radical with other carbon radicals or with hydrogen. During the crystal growth phase, reaction of carbon radicals (defective carbon or non 6-membered rings) with $\mathrm{H}$ leads to loss of reactivity and subsequent termination of the reaction (passivation of the defect site). An increase in $\mathrm{H}$ content leads to an increase in number of stabilized defective sites and formation of defective carbon nanostructures. On the other hand, in absence of hydrogen, the carbon radicals in the evolving crystal can react with other carbon radicals and undergo structural rearrangement to form 6-membered rings (thereby reducing the number of defects). Thus, carbon nanostructures formed in absence of hydrogen are less defective. 


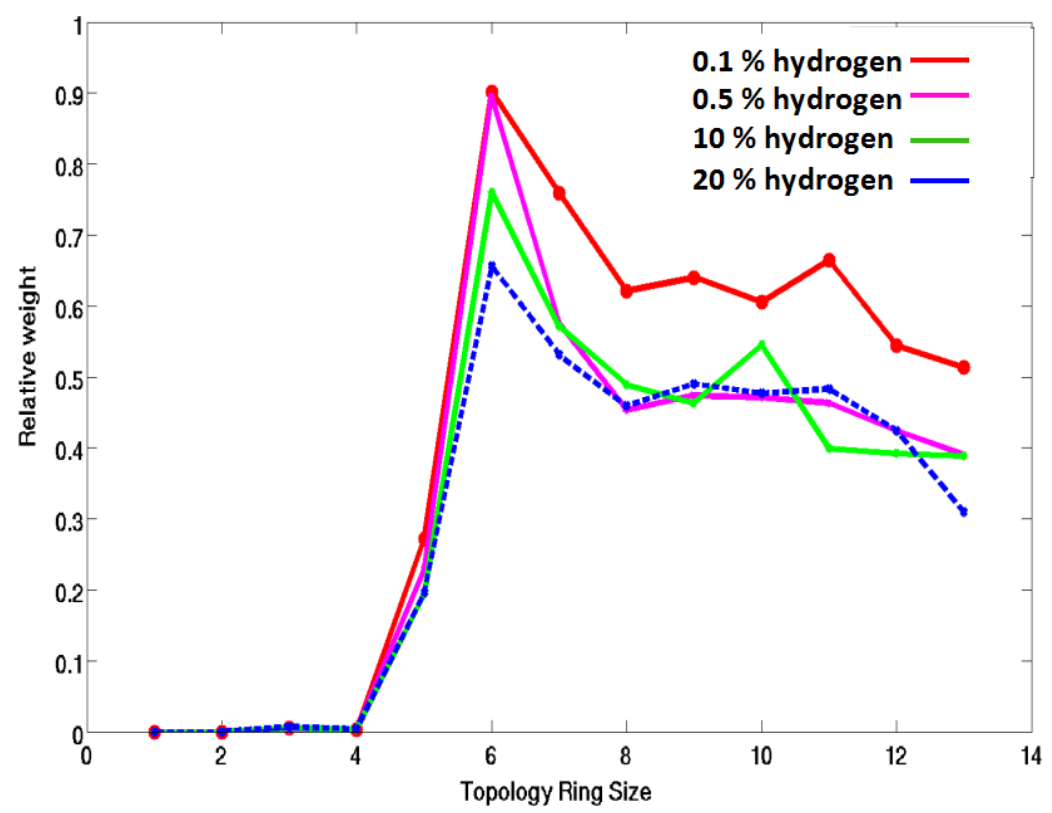

(a)

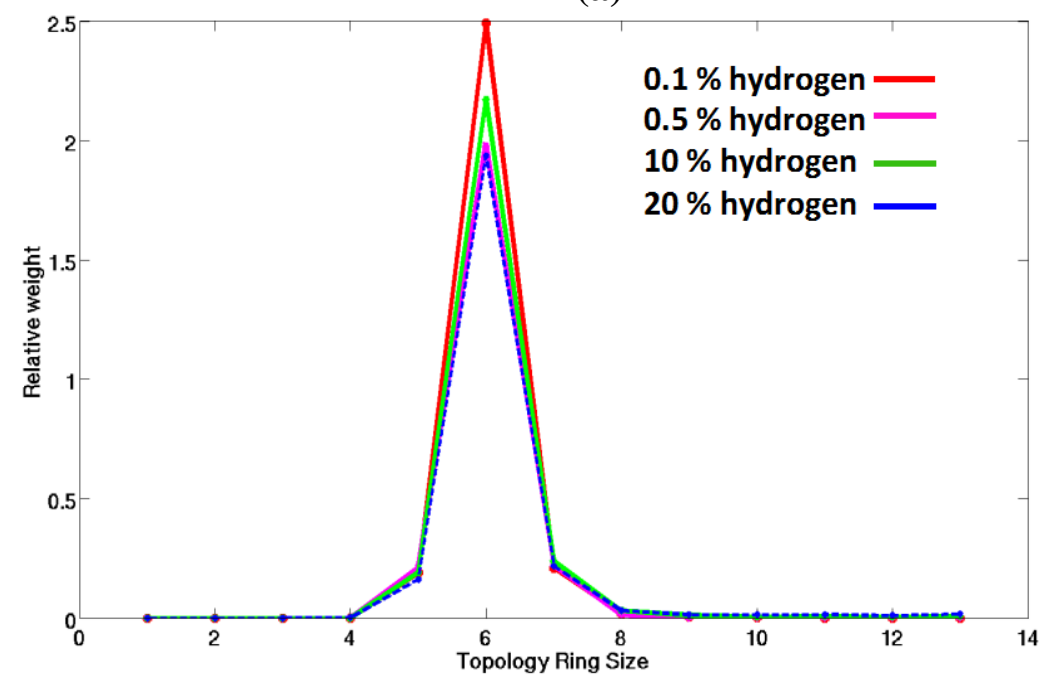

(b)

Figure 11: Graphitic order as determined by ring statistics for systems with various residual hydrogen content. The data is collected at the end of cooling process at $300 \mathrm{~K}$. Two different cooling rates are shown (a) $67 \mathrm{~K} / \mathrm{ps}$ (b) $1.34 \mathrm{~K} / \mathrm{ps}$.

\section{Conclusion}

Our simulations indicate that cooling rate strongly influences the long range ordering of carbon atoms in the final nanostructure via controlling the reaction pathways, and governs the 
formation of topological defects. At faster cooling rates $(>60 \mathrm{~K} / \mathrm{ps})$, the carbon atoms are not allowed sufficient time to diffuse, which results in quenching of the disordered configuration, as indicated by a continuous drop in configurational energy. Upon cooling at sufficiently slow rates $(<4 \mathrm{~K} / \mathrm{ps})$, the crystallization of carbon melts proceeds via growth of a few nuclei, causing changes in the slope of configurational energy drop with decrease in temperature. Our analysis of the topology of the obtained carbon nanostructures clearly demonstrates that a decrease in cooling rate progressively increases the long-range graphitic ordering of carbon nanostructures; for instance, at rates $<1.4 \mathrm{~K} / \mathrm{ps}$, ordered carbon onions comprising mainly of 6membered carbon rings form with few defects. This is consistent with peaks being observed at larger distances (beyond next-nearest neighbors) in the C-C RDFs at slow cooling rates; such peaks are non-existent at the fast rates. Furthermore, we find that the residual hydrogen has a profound effect on the long range and graphitic order at faster cooling rates. Finally, in accordance with these TARMD simulations, our experiments demonstrate that one can exercise control over the microstructural evolution of defects by tuning the cooling rate during the pyrolysis of PE. Thus, this work provides an effective technique to synthesis novel carbon nanostructures with tailored topological defects with tremendous promise in specific device applications.

\section{Acknowledgements:}

Use of the Center for Nanoscale Materials was supported by the U. S. Department of Energy, Office of Science, and Office of Basic Energy Sciences, under Contract No. DE-AC0206CH11357. This research used resources of the National Energy Research Scientific Computing Center, a DOE Office of Science User Facility supported by the Office of Science of the U.S. Department of Energy under Contract No. DE-AC02-05CH11231. An award of computer time was provided by the Innovative and Novel Computational Impact on Theory and Experiment (INCITE) program. This research used resources of the Argonne Leadership Computing Facility, which is a DOE Office of Science User Facility supported under Contract DE-AC02-06CH11357. 


\section{References:}

[1] Hansson A, Paulsson M, Stafström S. Effect of bending and vacancies on the conductance of carbon nanotubes. Physical Review B. 2000;62:7639.

[2] Ewels CP, Heggie MI, Briddon PR. Adatoms and nanoengineering of carbon. Chemical Physics Letters. 2002;351:178.

[3] Hashimoto A, Suenaga K, Gloter A, Urita K, Iijima S. Direct evidence for atomic defects in graphene layers. Nature. 2004;430:870.

[4] Su DS, Perathoner S, Centi G. Catalysis on nano-carbon materials: Going where to? Catalysis Today. 2012;186:1.

[5] Ma J, Alfè D, Michaelides A, Wang E. Stone-Wales defects in graphene and other planar sp2-bonded materials. Physical Review B. 2009;80:033407.

[6] Sammalkorpi M, Krasheninnikov A, Kuronen A, Nordlund K, Kaski K. Mechanical properties of carbon nanotubes with vacancies and related defects. Physical Review B. 2004; 70(24):245416.

[7] Charlier J-C, Ebbesen TW, Lambin P. Structural and electronic properties of pentagonheptagon pair defects in carbon nanotube. Physical Review B. 1996;53:11108.

[8] Peres NMR, Guinea F, Neto AHC. Electronic properties of disordered two-dimensional carbon. Physical Review B. 2006;73:125411.

[9] Lahiri J, Lin Y, Bozkurt P, Oleynik II, Batzil M. An extended defect in graphene as a metallic wire. Nature Nanotechnology. 2010;5:326.

[10] Sielemann R, Kobayashi Y, Yoshida Y, Gunnlaugsson HP, Weyer G. Magnetism at Single Isolated Iron Atoms Implanted in Graphite. Physical Review Letters. 2008;101:137206.

[11] López-Sancho MP, Juan Fd, Vozmediano MAH. Magnetic moments in the presence of topological defects in graphene. Physical Review B. 2009;79:075413.

[12] Mistry KK, Pol VG, Thackeray MM, Wen J, Miller DJ, Erdemir A. Synthesis and Tribology of Micro-Carbon Sphere Additives for Enhanced Lubrication. Tribology Transactions. 2015;58(3):474.

[13] Fiegl B, Kuhnert R, Ben - Chorin M, Koch F. Evidence for grain boundary hopping transport in polycrystalline diamond films Applied Physics Letters. 1994(65):371.

[14] Banhart F, Kotakoski J, Krasheninnikov AV. Structural Defects in Graphene. ACS Nano. 2011;5(1):26.

[15] Mendes RG, Bachmatiuk A, Büchner B, Cunibertibc G, Rümmeli MH. Carbon nanostructures as multi-functional drug delivery platforms. Journal of Materials Chemistry B. 2013;1:401.

[16] Lu AJ, Pan BC. Nature of Single Vacancy in Achiral Carbon Nanotubes. Physical Review Letters. 2004;92:105504.

[17] Nichols JA, Saito H, Deck C, Bandaru PR. Artificial introduction of defects into vertically aligned multiwall carbon nanotube ensembles: Application to electrochemical sensors. Journal of Applied Physics. 2007;102:064306.

[18] Nordlund K, Keinonen J, Mattila T. Formation of ion irradiation induced small-scale defects on graphite surfaces. Physical Review Letters. 1996;77:699.

[19] Krasheninnikov AV, Nordlund K, Sirviö M, Salonen E, Keinonen J. Formation of ionirradiation-induced atomic-scale defects on walls of carbon nanotubes. Physical Review B. 2001;63:245405. 
[20] Skákalová V, Kaiser AB, Dettlaff-Weglikowska U, Hrnčariková K, Roth S. Effect of Chemical Treatment on Electrical Conductivity, Infrared Absorption, and Raman Spectra of Single-Walled Carbon Nanotubes. The Journal of Physical Chemistry B. 2005;109(15):7174.

[21] Pol VG, Pol SV, Gedanken A. Dry Autoclaving for the Nanofabrication of Sulfides, Selenides, Borides, Phosphides, Nitrides, Carbides, and Oxides Advance Materials. 2011;23(10):1179 - 90.

[22] Deshmukh SA, Kamath G, Pol VG, Sankaranarayanan SKRS. Kinetic Pathways To Control Hydrogen Evolution and Nanocarbon Allotrope Formation via Thermal Decomposition of Polyethylene. Journal of Physical Chemistry C. 2014;118(18):9706.

[23] Pol VG, Wen J, Lau KC, Callear S, Bowron DT, Lin C-K, et al. Probing the evolution and morphology of hard carbon spheres. Carbon. 2014;68:2014.

[24] Pol VG, Thackeray MM. Spherical carbon particles and carbon nanotubes prepared by autogenic reactions: Evaluation as anodes in lithium electrochemical cells. Energy Environ Sci. 2011;4:1904 - 12.

[25] Pol SV, Pol VG, Gedanken A. Reactions under autogenic pressure at elevated temperature (RAPET) of various alkoxides: formation of metals/metal oxides-carbon core-shell structures. Chem-Eur J. 2004;10(18):4467 - 73.

[26] Pol VG, Motiei M, Gedanken A, Calderon-Moreno J, Yoshimura M. Carbon spherules: synthesis, properties and mechanistic elucidation. Carbon. 2004;42:111 - 6.

[27] Pol VG, Pol SV, Gedanken A. Dry Autoclaving for the Nanofabrication of Sulfides, Selenides, Borides, Phosphides, Nitrides, Carbides, and Oxides Adv Mater 2011;23:1179 - 90.

[28] Pol VG, Wen J, Lau KC, Callear S, Bowron DT, Lin C-K, et al. Probing the evolution and morphology of hard carbon spheres. Carbon. 2014;68:104 - 11.

[29] Pol VG, Thiyagarajan P. Remediating plastic waste into carbon nanotubes. J Environ Montior. 2010;12:455 - 9.

[30] Pol VG. Upcycling: Converting Waste Plastics into Paramagnetic, Conducting, Solid, Pure Carbon Microspheres. Environ Sci Technol 2010;44:4753 - 9.

[31] Pol SV, Pol VG, Sherman D, Gedanken A. A solvent free process for the generation of strong, conducting carbon spheres by the thermal degradation of waste polyethylene terephthalate. Green Chemistry. 2009;11(4):448-51.

[32] Pol VG, Thiyagarajan P. Measurement of Autogenous Pressure and Dissociated Species during the Thermolysis of Mesitylene for the Synthesis of Monodispersed, Pure, Paramagnetic Carbon Particles. Industrial \& Engineering Chemistry Research. 2009;48(3):1484-9.

[33] Wen J-G, Miller DJ, Zaluzec NJ, Hiller JM, Cook RE. Contribution of Cc-Correction to High-Resolution TEM at All Electron Energy Loss Regimes. Microscopy and Microanalysis; p. 594-5.

[34] Brenner DW, Shenderova OA, Harrison JA, Stuart SJ, Ni B, Sinnott SB. A secondgeneration reactive empirical bond order (REBO) potential energy expression for hydrocarbons Journal of Physics: Condensed Matter. 2002;14(4):783.

[35] Berman D, Deshmukh SA, Sankaranarayanan SKRS, Erdemir A, Sumant AV. Macroscale superlubricity enabled by gr aphene nanoscroll formation. Science. 2015:DOI: 10.1126/science. 1262024

[36] Allen MP, Tildesley DJ. Computer Simulation of Liquids: Oxford University Press; 1987. 
[37] Plimpton S. Fast Parallel Algorithms for Short-Range Molecular Dynamics. Journal of Computations Physics. 1995;117(1):1 - 19.

[38] Brenner DW, Shenderova OA, Harrison JA, Stuart SJ, Ni B, Sinnott SB. A secondgeneration reactive empirical bond order (REBO) potential energy expression for hydrocarbons J Phys: Condens Matter. 2002;14:783.

[39] Handbook of Chemistry \& Physics. ISBN 0-8493-0465-2: CRC Press.

[40] Deshmukh AA, Mhlanga SD, Coville NJ. Carbon spheres. Materials Science \& Engineering R-Reports. 2010;70(1-2):1-28.

[41] Inagaki M. Discussion of the formation of nanometric texture in spherical carbon bodies. Carbon. 1997;35(5):711-3.

[42] Donnet JB, Voet A. Carbon black. Dekker, 1993.

[43] Franklin R. Crystallite growth in graphitizing and non-graphitizing carbonsgr. Proceedings of Royal Society of London A. 1951;209:196-218.

[44] Rhim Y-R. The structure and property evolution of cellulose during carbonization. John Hopkins University, Doctor in Philosophy, 2009.

[45] Brooks JD, Taylor GH. New York: Dekker; 1968.

[46] Boukhvalov DW, Katsnelson MI. Chemical Functionalization of Graphene with Defects. Nano Letters. 2008;8(12):4373-9.

[47] Peng X, Ahuja R. Symmetry Breaking Induced Bandgap in Epitaxial Graphene Layers on SiC. Nano Letters. 2008;8(12):4464-8.

[48] Banhart F, Kotakoski J, Krasheninnikov AV. Structural Defects in Graphene. Acs Nano. 2011;5(1):26-41.

[49] Duplock EJ, Scheffler M, Lindan PJD. Hallmark of perfect graphene. Physical Review Letters. 2004;92(22).

[50] Bockhorn H, Hornung A, Hornung U, Schawaller D. Kinetic study on the thermal degradation of polypropylene and polyethylene. Journal of Analytical and Applied Pyrolysis. 1999;48:93.

[51] Ranzi E, Dente M, Faravelli T, Bozzano G, Fabini S, Nava R, et al. Kinetic modeling of polyethylene and polypropylene thermal degradation Journal of Analytical and Applied Pyrolysis 1997;40 - 41:305 - 19.

[52] Poutsma ML. Reexamination of the Pyrolysis of Polyethylene: Data Needs, FreeRadical Mechanistic Considerations, and Thermochemical Kinetic Simulation of Initial Product-Forming Pathways. Macromolecules 2003;36:8931.

[53] Poutsma ML. Evaluation of the Kinetic Data for Intramolecular 1,x-Hydrogen Shifts in Alkyl Radicals and Structure/Reactivity Predictions from the Carbocyclic Model for the Transition State. The Journal of Organic Chemistry. 2007;72(1):150 - 61.

[54] Ceamanos J, Mastral JF, Millera A, Aldea ME. Kinetics of pyrolysis of high density polyethylene. Comparison of isothermal and dynamic experiments. Journal of Analytical and Applied Pyrolysis. 2002;65(2):93 - 110.

[55] David DJ, Misra A. Relating Materials Properties to Structure with MATPROP Software Handbook and Software for Polymer Calculations and Materials Properties: CRC Press; 2001.

[56] Huheey JE, Keiter EA, Keiter RL. Inorganic Chemistry: Principles of Structure and Reactivity. 4th ed: New York: Harper Collins College Publishers 1993.

[57] Frenklach M. Reaction mechanism of soot formation in flames. Physical Chemistry Chemical Physics. 2002;4:2028 - 37. 
[58] Frenklach M, Wang H. Detailed Modeling of Soot Particle Nucleation and Growth. Symposium (International) on Combustion. 1991;23(1):1559 - 66.

[59] Bockhorn H. Soot Formation in Combustion Mechanisms and Models: Springer Series in Chemical Physics; 1994.

[60] Wal RLV, Tomasek AJ. Soot nanostructure: dependence upon s ynthesis conditions. Combustion and Flame. 2004;136(1 - 2):129 - 40.

[61] Xue K, Niu L-S, Shi H-J. Effects of quench rates on the short- and medium-range orders of amorphous silicon carbide: A molecular-dynamics study J Appl Phys. 2008; 104:053518.

[62] Yuan X, Cormack AN. Efficient algorithm for primitive ring statistics in topological networks. Comput Mater Sci. 2002;24:343 - 60.

[63] Tsefrikas VM, Scott LT. Geodesic polyarenes by flash vacuum pyrolysis. Chemical Reviews. 2006;106:4868. 